\title{
Spectral algorithm for non-destructive damage localisation: Application to an ancient masonry arch model
}

\author{
Maria-Giovanna Masciotta a,*, Luís F. Ramos ${ }^{a}$, Paulo B. Lourenço ${ }^{a}$, \\ Marcello Vasta ${ }^{\mathrm{b}}$ \\ a ISISE, University of Minho, Department of Civil Engineering, Campus de Azurém, 4800-058 Guimarães, Portugal \\ b University “G.d'Annunzio”, Department of Engineering and Geology, 65127 Pescara, Italy
}

\section{A R T I C L E I N F O}

\section{Article history:}

Received 10 March 2016

Received in revised form

13 June 2016

Accepted 20 June 2016

Available online 26 July 2016

\section{Keywords:}

Non-destructive damage localisation

Eigenvalue decomposition

Spectral-based method

Historical structures

Masonry arch

\begin{abstract}
A B S T R A C T
Structural monitoring and vibration-based damage identification methods are fundamental tools for condition assessment and early-stage damage identification, especially when dealing with the conservation of historical constructions and the maintenance of strategic civil structures. However, although the substantial advances in the field, several issues must still be addressed to broaden the application range of such tools and to assert their reliability. This study deals with the experimental validation of a novel method for non-destructive damage identification purposes. This method is based on the use of spectral output signals and has been recently validated by the authors through a numerical simulation. After a brief insight into the basic principles of the proposed approach, the spectral-based technique is applied to identify the experimental damage induced on a masonry arch through statically increasing loading. Once the direct and cross spectral density functions of the nodal response processes are estimated, the system's output power spectrum matrix is built and decomposed in eigenvalues and eigenvectors. The present study points out how the extracted spectral eigenparameters contribute to the damage analysis allowing to detect the occurrence of damage and to locate the target points where the cracks appear during the experimental tests. The sensitivity of the spectral formulation to the level of noise in the modal data is investigated and discussed. As a final evaluation criterion, the results from the spectrum-driven method are compared with the ones obtained from existing non-model based damage identification methods.
\end{abstract}

(c) 2016 Elsevier Ltd. All rights reserved.

\section{Introduction}

In the last decades the growing interest concerning the conservation of built cultural heritage as well as the maintenance and repair of existing civil structures have been drawing the attention of the engineering community to the possibility of identifying damage at the earliest possible stage. The relevance of such an issue lies in practical reasons, primarily avoiding the impairment of value and the normal function of structures that are valid from a cultural or strategic point of view and for economic and political reasons. The evaluation of the structural conditions is needed to plan cost-effective remedial measures, before the extension of damage leads the system to stop operation or requires expensive in-depth interventions.

\footnotetext{
* Corresponding author.

E-mail addresses: mg.masciotta@gmail.com (M.-G. Masciotta), lramos@civil.uminho.pt (L.F. Ramos), pbl@civil.uminho.pt (P.B. Lourenço), mvasta@unich.it (M. Vasta).
} 
However, restrictions in the inspection and the removal of specimens in buildings of historical value, as well as the high costs involved in inspection and diagnosis, often result in limited information about the internal constructive system of the structural elements or the properties of existing materials [1]. Nowadays, the availability of inverse approaches contributes to overcome this problem allowing to characterize the 'unknown' parameters of a structure by the identification of its dynamic properties. Moreover, the direct interdependence between dynamic and physical properties makes modal-based methods suitable tools for detecting the structural damage. For the purpose of this work, damage can be considered as an adverse condition that compromises the structural integrity of a system and affects its performance in terms of load-bearing capacity of the structural elements, materials strength, stiffness, etc. The alterations induced into the system by the damage and the reversibility of the process depend on the severity and size of the damage and last, but not least, on the number and type of elements involved. Implicit is that the stiffness degradation due to the loss of structural integrity will be reflected in changes of the properties of the system, notably its modal parameters (frequencies, mode shapes and damping ratios). The possibility to easily measure these quantities has been fostering their use as damage indicators in all engineering fields [2], from civil structures to offshore systems [3], from composite materials [4] to masonry-like constructions [5]. Among the damage identification methods based on measured vibration responses [6], the ones combining frequencies, mode shapes and/or modal curvatures are usually more successful since they allow to detect the presence of damage in a global way and they also provide spatial information to pinpoint it. Non-modal-based indexes for non-destructive damage detection purposes have been proposed [7,8]: in this case, the innovative aspect is the use of statistical properties of random signals in order to attenuate measurement uncertainties so as to avoid ill-conditioned outcomes. Nevertheless, none of the methods currently available in literature seems to give accurate results for all levels of damage and for all types of structural systems [9]. Several issues must still be addressed to make these powerful techniques efficient tools applicable to a variety of cases, e.g.: the level of sensitivity of the modal parameters in catching micro-cracks in a structure and detecting damage still not visible to human eyes; the performance of the method when dealing with a limited number of measurements or with excitations whose frequency content is narrow-banded; the capability of distinguishing between changes in modal parameters due to environmental conditions from the ones due to the occurrence of damage; the accuracy of the method when handling noisy data and the possibility of reducing the noise influence in the measurements. These critical issues call for further research in the field.

In the light of what was stated above, the aim of the present paper is to provide a robust formulation for non-destructive damage identification purposes based on the use of spectral analysis methods. The main contribution of this approach is the possibility to accurately identify the damage affecting a structure only from the knowledge of the second order properties of measured output vibration signals. In this regard, an in-depth analysis of evolutionary spectral quantities extracted from the system's output power spectrum matrix over different damage scenarios is performed. The efficiency and reliability of the approach is validated firstly by direct application to an old masonry arch replica subjected to progressive damage in laboratory environment and then by comparison with other Vibration-Based Damage Identification Methods (VBDIMs) applied to the same data sets. The spectral response signals necessary to start the procedure are computed from the nodal processes acquired by output-only identification techniques and the modal information are estimated from the eigenparameters decomposition of the output Power Spectral Density (PSD) matrix. The spectral modes belonging to different structural conditions are progressively compared to identify the damage by means of an index that combines extracted eigenvectors and eigenvalues. It is shown that the eigenvectors alone are not sufficient to localise the damage if not properly weighed through their corresponding eigenvalues. With this purpose, a Broad-Band (BB) damage index and a Narrow-Band (NB) damage index are computed and compared in order to analyse the sensitivity of the algorithm to the frequency range considered. Narrowing the damage index frequency bandwidth is shown to be the best approach to reduce the influence of noise in the modal data, avoiding likely ill-conditioned results.

The next sections of the paper are organised as follows: Section 2 gives a concise overview of the principles underlying the spectral method, explains how the spectral damage algorithm is derived and details the main steps of the procedure; Section 3 describes the experimental campaign used as benchmark; Section 4 is devoted to the spectrum-driven damage analysis of the masonry arch, whose results are extensively discussed in Section 5 together with the comparison between the spectral damage index and other vibration-based damage indexes; finally, Section 6 gives a summary of the work and the main conclusions.

\section{Spectrum-driven damage identification method}

\subsection{Basic principles}

In stochastic environment, the response $\mathbf{X}(t)$ of a system to a dynamic loading $\mathbf{F}(t)$ can be defined as unidimensional multivariate stochastic vector process whose elements are the time-dependent nodal response processes. In this context, the dynamic characterisation of a given structural system can be performed through the application of normal spectral analysis methods based on the eigenvalue decomposition of the response Power Spectral Density (PSD) matrix $\mathbf{S}_{x}(\omega)$, according to the following expression:

$$
\mathbf{S}_{X}(\omega)=\Psi_{X}(\omega) \boldsymbol{\Lambda}_{X}(\omega) \Psi_{X}^{H}(\omega)
$$


where $\boldsymbol{\Lambda}_{X}(\omega)=\operatorname{diag}\left\{\lambda_{1}(\omega), \lambda_{2}(\omega), \ldots \ldots, \lambda_{j}(\omega), \ldots \ldots \lambda_{n}(\omega)\right\}$ is a diagonal matrix including frequency-dependent eigenvalues in decreasing order and $\boldsymbol{\Psi}_{X}(\omega)=\left[\boldsymbol{\Psi}_{1}(\omega) \boldsymbol{\Psi}_{2}(\omega) \ldots \boldsymbol{\Psi}_{j}(\omega) \ldots \boldsymbol{\Psi}_{n}(\omega)\right]$ is a complex matrix consisting of mutually orthogonal coordinate-dependent eigenvectors, i.e. $\Psi_{X}(\omega) \mathbf{\Psi}_{X}^{H}(\omega)=\mathbf{I}$, being $\boldsymbol{\Psi}_{X}^{H}(\omega)$ the complex conjugate transpose of $\boldsymbol{\Psi}_{X}(\omega)$. Each eigenvalue $\lambda_{i}(\omega)$ is proportional to the energy of a certain vibration mode, whereas each eigenvector $\boldsymbol{\Psi}_{i}(\omega)$ is a mode shape estimation corresponding to a certain eigenvalue. Note that, as the eigenvalues are decreasingly ordered $\left(\lambda_{i}=1 / \omega_{i}^{2}\right)$, more importance is given to the lower modes, which are the modes featuring greater energy of vibration and are experimentally estimated with less uncertainty. Moreover, since $\mathbf{S}_{X}(\omega)$ is a square Hermitian matrix, all eigenvalues are nonnegative, $\lambda_{i}(\omega)$ $\geq 0$, meaning that they coincide with the singular values of the matrix, as well as the eigenvectors $\boldsymbol{\Psi}_{j}(\omega)$ coincide with the singular vectors [21].

The key assumption of the spectral analysis lies in the consideration of a constant power spectral density for the input force, i.e. $\mathbf{S}_{F}(\omega)=\mathbf{C}$ (white noise assumption). In this way, the modal properties of the structural system can be directly estimated from the power spectrum matrix of the response processes:

$$
\mathbf{S}_{X}(\omega)=\mathbf{H}(\omega) \mathbf{S}_{F}(\omega) \mathbf{H}^{H}(\omega)=\mathbf{H}(\omega) \mathbf{C H}^{H}(\omega)
$$

However, it has been proved elsewhere [23] that the method is also suitable when dealing with input forces other than white-noise excitations. A complete description of the fundamentals of stochastic dynamics and spectral analysis is not pursued in this study and for this purpose the reader is referred to text books $[10,11,20]$. Main goal of the present work is to describe a novel spectral-based approach for damage identification with application to experimental results.

\subsection{Derivation of the spectral damage index}

The spectral-based damage identification method stems from the consideration that changes in the system's stiffness caused by evolutionary damage scenarios are reflected by changes in the system's response power spectrum matrix as well as its eigenparameters. It has been shown [22] that a multivariate stochastic vector process $\mathbf{X}(t)$ with power spectrum matrix $\mathbf{S}_{X}(\omega)$ can be expressed as a summation of $n$ independent fully coherent stochastic processes as follows:

$$
\mathbf{X}(t)=\sum_{k=1}^{n} \mathbf{Y}_{k}(t)
$$

where:

$$
\mathbf{Y}_{k}(t)=2 \sqrt{\Delta \omega} \operatorname{Re}\left(\sum_{j=1}^{n} \boldsymbol{\Psi}_{k}\left(\omega_{j}\right) \sqrt{\lambda_{k}\left(\omega_{j}\right)} \cdot P_{j}^{(k)} e^{i \omega_{j} t}\right)
$$

in which $\boldsymbol{\Psi}_{k}(\omega)$ and $\lambda_{k}(\omega)$ are the $k$ th eigenvector and eigenvalue of $\boldsymbol{S}_{X}(\omega), \operatorname{Re}[\bullet]$ returns the real part of the complex value expression and $P_{j}^{(k)}$ is a random complex number defined as shown below:

$$
P_{j}^{(k)}=R_{j}^{(k)}-i I_{j}^{(k)}
$$

$R_{j}^{(k)}$ and $I_{j}^{(k)}$ being zero-mean normal random numbers obeying the following orthogonality relationships:

$$
E\left[R_{j}^{(r)} R_{j}^{(s)}\right]=\frac{1}{2} \delta_{j k} \delta_{r s} \quad E\left[I_{j}^{(r)} I_{j}^{(s)}\right]=\frac{1}{2} \delta_{j k} \delta_{r s} \quad E\left[R_{j}^{(r)} I_{j}^{(s)}\right]=0
$$

and $\delta$ being the Kronecker delta $\left(\delta_{j k}=1\right.$ if $j=k, \delta_{j k}=0$ if $j \neq k$ ).

Given a damaged multivariate stochastic vector process $\mathbf{X}^{d}(t)$, it has been demonstrated [23] that the difference between the two vector processes $\mathbf{X}^{d}(t)$ and $\mathbf{X}(t)$ can be expressed as follows:

$$
\begin{aligned}
& \Delta \mathbf{X}(t)=\mathbf{X}^{d}(t)-\mathbf{X}(t)=\sum_{k=1}^{m}\left(\mathbf{Y}_{k}^{d}(t)-\mathbf{Y}_{k}(t)\right) \\
& \Delta \mathbf{X}(t)=2 \sqrt{\Delta \omega} \operatorname{Re}\left(\sum_{k=1}^{m} \sum_{j=1}^{n} \Delta \boldsymbol{\Psi}_{k}\left(\omega_{j}\right) \cdot P_{j}^{(k)} e^{i \omega_{j} t}\right)
\end{aligned}
$$

where:

$$
\Delta \boldsymbol{\Psi}_{k}\left(\omega_{j}\right)=\boldsymbol{\Psi}_{k}^{d}\left(\omega_{j}\right) \sqrt{\lambda_{k}^{d}\left(\omega_{j}\right)}-\boldsymbol{\Psi}_{k}\left(\omega_{j}\right) \sqrt{\lambda_{k}\left(\omega_{j}\right)}
$$

From the equations above it is deduced that the difference between a reference and a damage multivariate stochastic vector process depends on the eigenvectors and eigenvalues in which the PSD matrix of each process is decomposed. The complex function $\Delta \boldsymbol{\Psi}_{k}\left(\omega_{j}\right)$ acts as a natural damage indicator: when $\Delta \boldsymbol{\psi}_{k}\left(\omega_{j}\right)=0$ for any $k$ and $j$, then $\Delta \mathbf{X}(t)=\mathbf{X}^{d}(t)-\mathbf{X}(t)=0$, meaning that no damage occurs in the structure, i.e. $\mathbf{X}^{d}(t)=\mathbf{X}(t)$. In light of these consideration, the following spectral 
damage index has been defined [23]:

$$
\Delta \boldsymbol{\psi}=\sum_{k=1}^{m}|| \sum_{j=1}^{n}\left[\boldsymbol{\Psi}_{k}^{d}\left(\omega_{j}\right) \sqrt{\lambda_{k}^{d}\left(\omega_{j}\right)}\right]|-| \sum_{j=1}^{n}\left[\boldsymbol{\psi}_{k}^{u}\left(\omega_{j}\right) \sqrt{\lambda_{k}^{u}\left(\omega_{j}\right)}\right] \mid
$$

where $n$ indicates the frequency range, $m$ represents the eigenvector number and upper scripts $d$ and $u$ stand for damaged and undamaged conditions, respectively. The index $\Delta \boldsymbol{\psi}$ in Eq. (10) is based on the sum of the differences between the spectral modes of the damaged and undamaged structural conditions, where the spectral modes are estimated through the amplification of the eigenvectors extracted from the response PSD matrix by the square root of their corresponding eigenvalues $\left(\boldsymbol{\Psi}_{k}\left(\omega_{j}\right) \sqrt{\lambda_{k}\left(\omega_{j}\right)}\right)$. If no damage occurs in the structure, the index $\Delta \boldsymbol{\psi}$ in Eq. (10) is equals to zero since $\left|\sum_{j=1}^{n} \boldsymbol{\Psi}_{k}^{d}\left(\omega_{j}\right) \sqrt{\lambda_{k}^{d}\left(\omega_{j}\right)}\right|=\left|\sum_{j=1}^{n} \boldsymbol{\Psi}_{k}^{u}\left(\omega_{j}\right) \sqrt{\lambda_{k}^{u}\left(\omega_{j}\right)}\right|$. On the contrary, if damage occurs, the index $\Delta \boldsymbol{\psi}$ will be different than zero and will result in a vector of scalars (each one associated with a measured DOF) whose magnitude enables to identify the damage position in the system and to give a qualitative estimate of the damage size. For a comprehensive description of the spectral formulation the reader is referred to [23].

In order to study the sensitivity of the spectral damage identification algorithm to the frequency bandwidth considered, two different new spectral indexes are defined in the present work: a Broad-Band (BB) index and a Narrow-Band (NB) index. The $\mathrm{BB}$ index is computed by weighing eigenvalues and eigenvectors over the whole frequency domain of interest $\left\{\omega_{j} \mid\right.$ $\left.1 \leq \omega_{j} \leq n\right\}$; whereas the NB index is computed over a limited range of frequencies in the vicinity of the eigenvalues local maxima $\left\{\omega_{j} \mid \omega_{1} \leq \omega_{j} \leq \omega_{2}\right\}$. It is noted that the narrow-band index refers to frequencies which are not corrupted by the noise content present in the measurement data. Beyond this range, the frequency response of the system may result whether meaningless or ill-defined if the signal-to-noise ratio is low. Therefore, depending on the actual level of noise, the frequency bandwidth of the NB index can be infinitesimally narrowed as lower and upper boundary frequencies $\left(\omega_{1}, \omega_{2}\right)$ approach to the eigenfrequency $\omega_{j}$, viz. the resonant frequency of the system. Accordingly, Eq. (10) turns into:

$$
\Delta \boldsymbol{\psi}=\sum_{k=1}^{m}|| \boldsymbol{\psi}_{k}^{d}\left(\omega_{j}\right) \sqrt{\lambda_{k}^{d}\left(\omega_{j}\right)}|-| \boldsymbol{\psi}_{k}^{u}\left(\omega_{j}\right) \sqrt{\lambda_{k}^{u}\left(\omega_{j}\right)}||
$$

where $\omega_{j}=\omega_{1}, \omega_{2}, \ldots, \omega_{n}$ are the eigenfrequencies of the structure. As a result, the index $\Delta \boldsymbol{\psi}$ in Eq. (11) is based on the difference between the 'resonant spectral modes' of the damaged and undamaged configurations.

\subsection{Description of the method}

The damage identification methods can be categorized according to various criteria, i.e. the effect of damage on the structure or the level of damage provided $[6,12,13,14]$. As stated in the introduction, the levels of damage investigated in the present work are three: detection, localisation and assessment. Note that the third level of identification is treated only from a qualitative point of view, since the relationship between qualitative and quantitative damage measures has not been addressed. The adopted approach aims at identifying the damage of a masonry arch by the spectral analysis of its output signals, starting from the decomposition of the PSD matrix of the nodal responses in real eigenvalues and complex eigenvectors and proceeding with the progressive comparison between spectral modes of successive damage scenarios. A necessary condition to start the investigation procedure is the presence of at least two different scenarios, e.g. damaged and undamaged. The fundamental steps composing the method are presented hereafter.

\subsubsection{Damage detection}

The first step of the spectral-based approach involves the analysis of the global dynamic characteristics of the system, i.e. frequency and damping coefficient, in order to detect the presence of damage. Unlike natural frequencies, damping coefficients are very difficult to estimate accurately. Because of that, the addressed spectral method only uses frequency values as global parameters for damage detection purposes. The identification of these parameters is obtained for each scenario by the diagonalization of the system's spectral density matrix, known as eigenvalues plotting. Each eigenvalue $\lambda_{i}(\omega)$ is proportional to the energy of a certain vibration mode at a certain frequency, thus all the local maxima yielded by the eigenvalues curves allow to estimate the eigenfrequency (and the physical magnitude) of each vibration mode of the system. Following the evolution of these parameters during the dynamic tests and considering that frequency changes imply changes in the physical properties of the system (i.e. stiffness or flexibility) and vice versa, it is possible to keep under control the structural conditions and progressively detect the occurrence of damage.

\subsubsection{Damage localisation and assessment}

The second step of the procedure concerns the localisation of the structural damage. For this purpose, changes in the complex eigenvectors obtained from the PSD matrix decomposition are taken into consideration. Each eigenvector $\boldsymbol{\Psi}_{i}(\omega)$ is a mode shape estimate, viz. a coordinate-dependent modal parameter which can provide spatial information to pinpoint the damage. The number of eigenvectors equals the rank of the matrix, or rather the number of independent measured DOFs. The sum of all eigenvectors characterised by the same eigenfrequency gives the resonant eigenvectors of the structure. The 


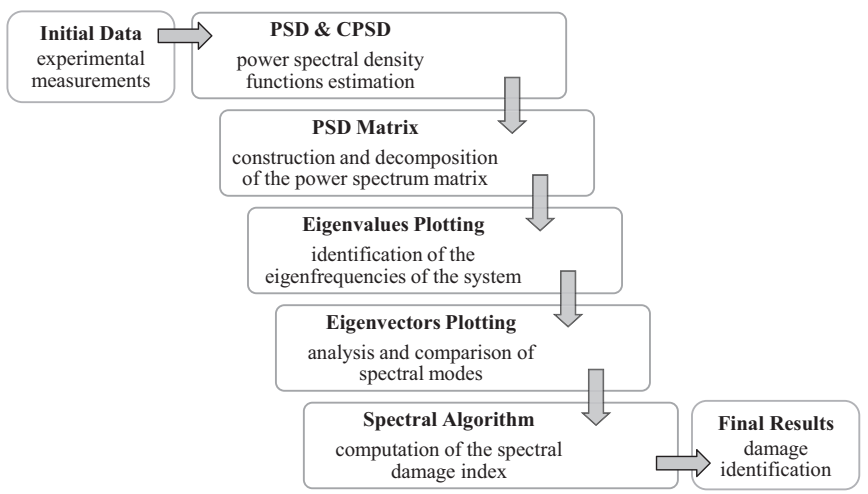

Fig. 1. Flowchart of the spectral-based damage identification method (PSD and CPSD indicate direct and cross power spectral density functions, respectively).

comparison between single resonant eigenvectors $\boldsymbol{\psi}_{i}(\omega)$ belonging to different Damage Scenarios (DSs) may help to locate the damage, but it is not sufficient without considering the spectral modes as a whole, i.e. $\boldsymbol{\psi}_{i}(\omega) \lambda_{i}(\omega)$, meaning that the energy of vibration associated with each eigenvector and weighed through its relevant eigenvalue must be accounted for. It is also stressed that eigenvectors are complex and so are the spectral modes. This comes up with the necessity to sort out the real part of each eigenvector from its imaginary part or to weigh both of them by considering the module. One of the main issue addressed in this work is indeed the analysis of these complex parameters in order to understand which part, whether the real or the imaginary, is the most relevant for damage identification. Although studies have shown that estimates of mode shapes can be obtained from the peak values of the imaginary part of FRFs for displacement or acceleration responses and from the peak values of the real part of FRFs for velocity responses [15], this issue is not so straightforward when dealing with spectral modes and still needs to be addressed properly.

The last step of the method consists in the computation of the spectral damage index defined in Eq. (10). Based on the combination of both eigenvectors and eigenvalues, this index permits not only to locate the damage but also to give a rough estimate of the damage size in the target points. As shown in Section 2.2, depending on the level of noise present in the measurements, the index can be computed whether weighing the eigenparameters over the whole frequency domain or narrowing the frequency bandwidth of interest, up to considering only resonant eigenvectors and eigenvalues. In case of multiple damage scenarios, a relative index can be calculated to progressively follow the evolution of damage. Fig. 1 shows the flowchart of the main steps of the adopted method.

\section{A masonry arch as case study}

Benchmark of the present work is the experimental campaign carried out in 2006 on a masonry arch built in the Structural Laboratory of the Civil Engineering Department of the University of Minho (Guimarães, Portugal) [9,16]. The arched structure was progressively damaged by statically increasing loading and, after each load stage (damage scenario), the dynamic response of the system was measured by means of output-only identification techniques. The spectrum-driven method for damage identification detailed makes use of the data coming from the dynamic identification tests performed on the arch model for a direct comparison with experimental results.

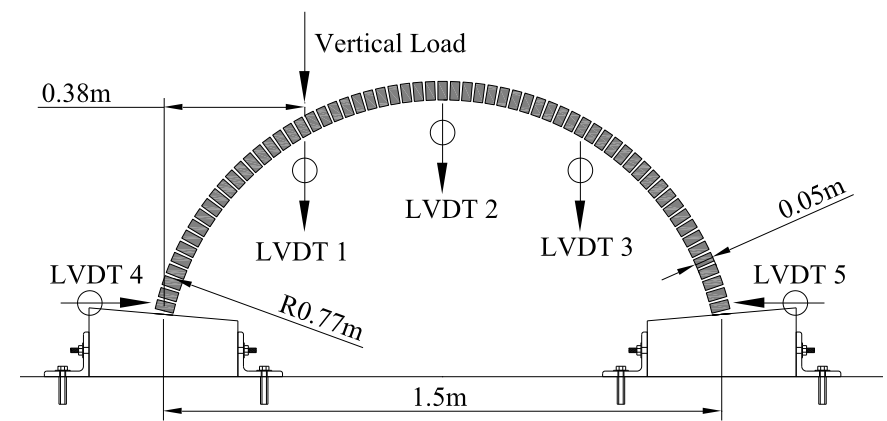

(a)

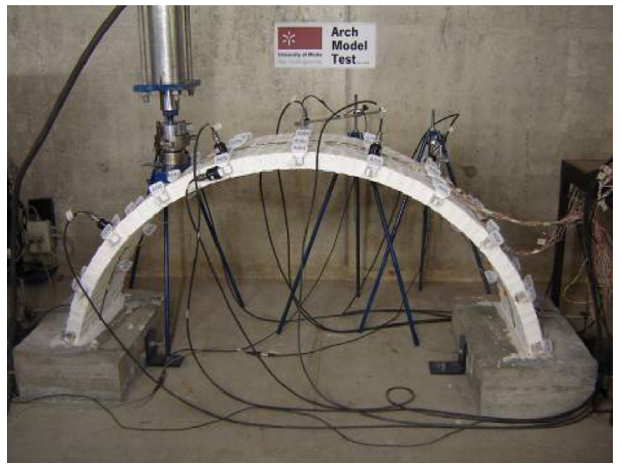

(b)

Fig. 2. Arch model: (a) geometry (front view) with location of the LVDTs during the static tests; (b) view of the test apparatus [9]. 


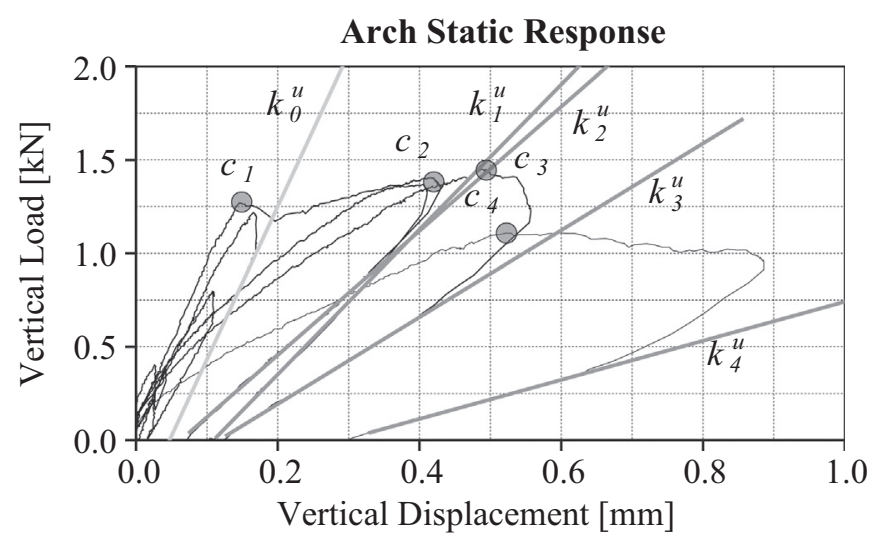

Fig. 3. Arch static response over the eight DSs [9].

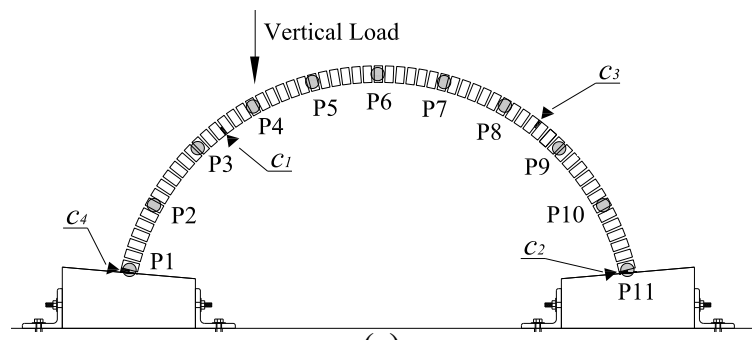

(a)

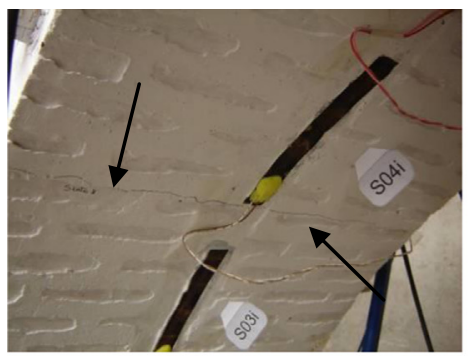

(c)

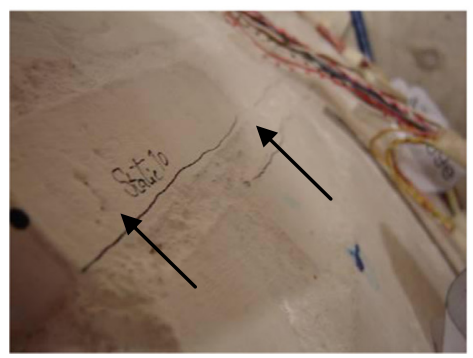

(e)

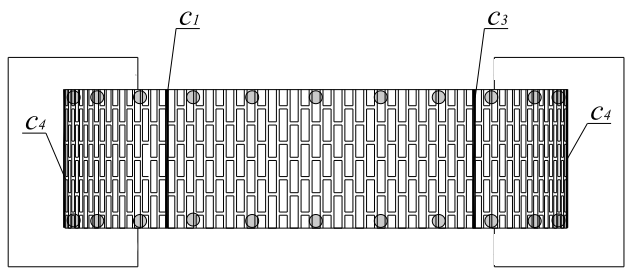

(b)

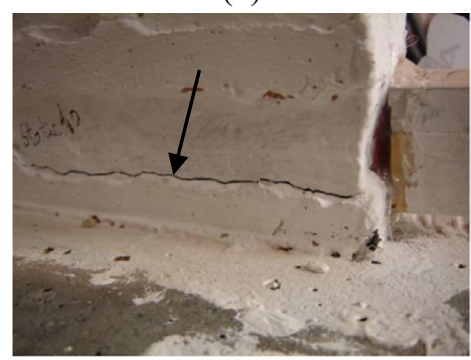

(d)

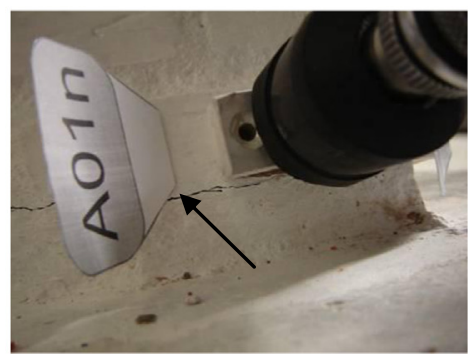

(f)

Fig. 4. Crack pattern, denoted as $c_{i}$, with $i=1,4$ : (a) and (b) crack location; (c) $\operatorname{crack} c_{1}$; (d) $\operatorname{crack} c_{2}$; (e) $\operatorname{crack} c_{3}$; (f) crack $c_{4}$ [9].

\subsection{Description of the arch}

The arch was built with low compressive strength clay bricks $\left(100 \times 50 \times 25 \mathrm{~mm}^{3}\right)$ and low quality mortar joints $(0.5 \mathrm{~cm}$ thickness) so as to be representative of the ancient masonry arches used in historical constructions. The geometrical features of the arch were the followings: semi-circular shape with $0.77 \mathrm{~m}$ radius, span of $1.50 \mathrm{~m}$, width equals to $0.45 \mathrm{~m}$ and thickness of $0.05 \mathrm{~m}$ (Fig. 2). The structure rested on two concrete abutments fixed on the floor by bolts. Detailed information about the construction technique and the mechanical properties of the materials are given in [16] and [17]. 


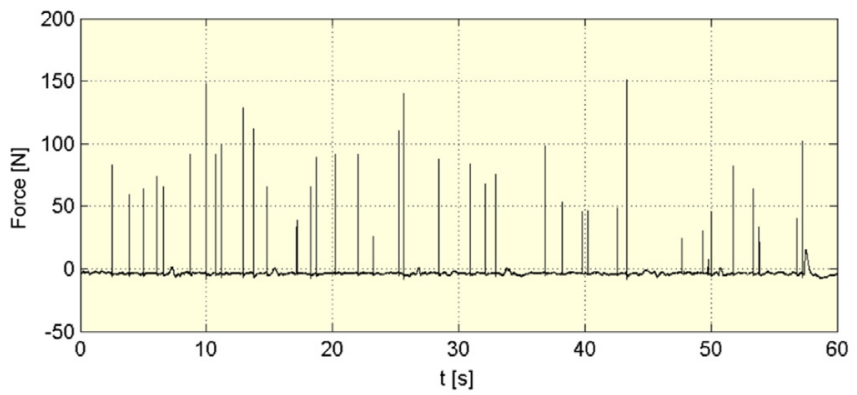

(a)

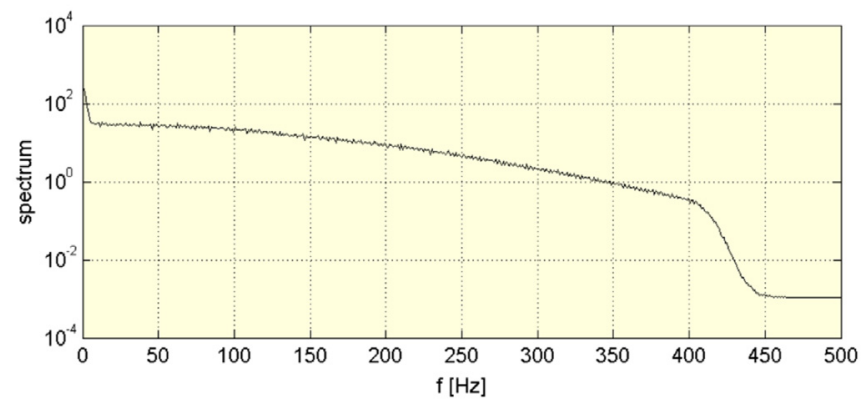

(b)

Fig. 5. Example of random impact excitation for one dataset: (a) time history and (b) power spectrum.

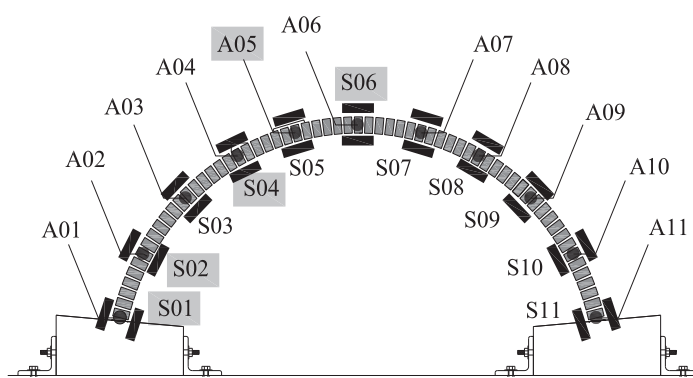

(a)

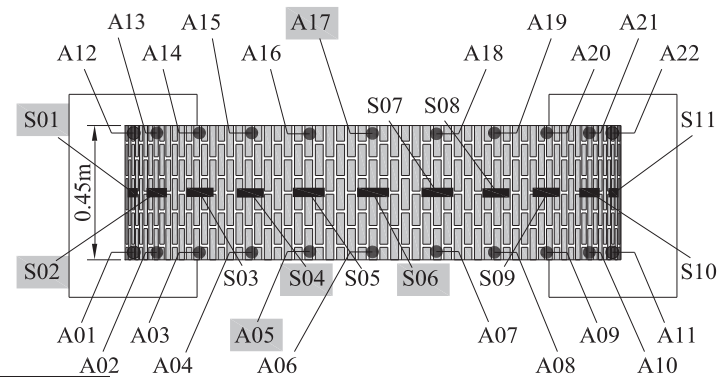

(b)

Fig. 6. Location of the measuring points for the dynamic tests: front (a) and top (b) views $\left(A_{i}\right.$ and $S_{i}$ denote accelerometers and strain gauges, respectively; reference sensors are indicated in grey boxes) [9].

Table 1

Statistics of the global modal parameters identified for the reference scenario.

\begin{tabular}{lllllll}
\hline Modes & $f(\mathrm{~Hz})$ & $\begin{array}{l}\sigma_{f} \\
(\mathrm{~Hz})\end{array}$ & $\mathrm{CV}_{f}(\%)$ & $\xi(\%)$ & $\sigma_{\xi}(\%)$ & $\mathrm{CV}_{\xi}(\%)$ \\
\hline 1 & 35.21 & 0.12 & 0.33 & 0.51 & 0.08 & 15.94 \\
2 & 66.58 & 0.36 & 0.55 & 0.64 & 0.09 & 0.14 \\
3 & 71.16 & 0.21 & 0.30 & 0.79 & 0.10 & 19.21 \\
4 & 124.52 & 0.74 & 0.60 & 0.73 & 0.22 & 12.32 \\
5 & 138.94 & 0.90 & 0.49 & 2.55 & 0.30 & 30.11 \\
6 & 172.54 & 0.84 & 1.08 & & 0.94 & 36.98 \\
\hline
\end{tabular}

\subsection{Experimental campaign}

\subsubsection{Static damage tests}

With the purpose of inducing progressive damage, a series of static tests were carried out on the arched structure to reach different Damage Scenarios (DSs). In total the load was applied using increment load levels, in a total of eight DSs (without including the original undamaged configuration). The test apparatus consisted of a hydraulic jack set at a quarter of 


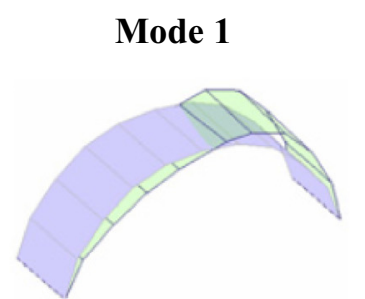

$35.21 \mathrm{~Hz}$

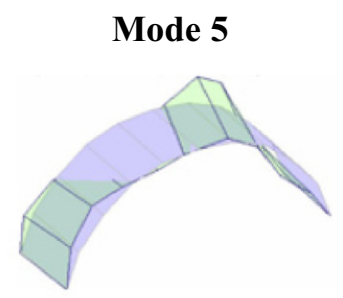

$138.49 \mathrm{~Hz}$

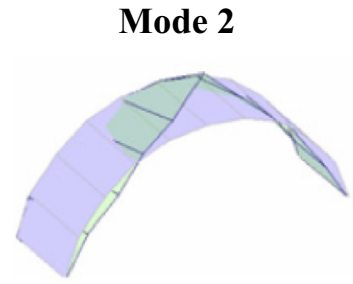

$66.58 \mathrm{~Hz}$

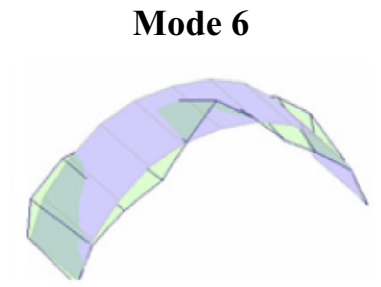

$172.54 \mathrm{~Hz}$

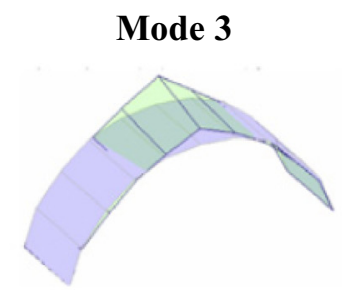

$71.16 \mathrm{~Hz}$

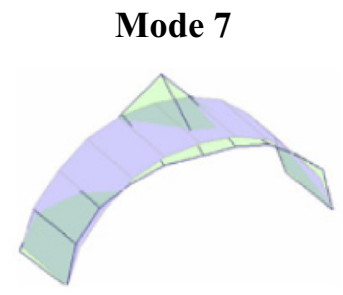

$196.76 \mathrm{~Hz}$

Fig. 7. Configuration of the vibration modes of the arch for the reference scenario [9].

the span to apply statically increasing loads and five LVDTs symmetrically placed along the arch to measure its response along the testing (Fig. 2). In order to replicate real situations of temporary loadings, such as vehicle or truck live loads, earthquakes or impact forces, the load was removed after each DS. Unloading resulted in crack closure, which makes the damage identification task further challenging. Four cracks progressively appeared during the static tests as clearly indicated by the loss of stiffness in the load-displacement diagram (Fig. 3), but all cracks became visible only in the loading path of the last damage scenario $\mathrm{DS}_{\mathrm{VIII}}$, with the exception of the first crack that was visually detected during the loading branch of DS The resulting crack pattern in the arch is presented in Fig. 4, where the measurement points $\mathrm{P}$ corresponding to the instrumented parts of the arch are indicated (the sensors location is detailed hereafter). Note that the designation $c_{1}$ to $c_{4}$ is random since the full crack sequence was impossible to detect and so was the load value corresponding to each crack. The crack locations are as follows: crack $c_{1}$ appeared at the intrados between positions P3 and P4 (but not beneath the load application point); crack $c_{2}$ was detected at the right support of the arch, corresponding to position P11; crack $c_{3}$ appeared at the extrados between positions P8 and P9; and crack $c_{4}$ was located at the left support of the arch, corresponding to position P1. The complete description of the static response of the arch for each single DS is presented in [16].

\subsubsection{Dynamic identification tests}

In order to follow the evolution of the modal properties of the masonry arch along the testing and to check its stiffness degradation with increasing damage, dynamic identification tests were performed after each static test. To ensure a fairly high signal-to-noise ratio, a random impact source was used to excite the system. This type of input was induced by a hammer of $2.5 \mathrm{~kg}$ of mass hitting the structure randomly in time and space so as to produce an excitation that could well approximate a multivariate white noise stochastic process. Indeed, the white noise can be defined as a random signal consisting of serially uncorrelated random variables with zero mean, finite variance and a constant power spectral density. In the present case, since the number and position of impulses are not correlated, the exogenous input conceivably tends to a multivariate stationary white noise stochastic process [24,25]. This is confirmed by analysing the time history of the force used to excite the arch along with the corresponding power spectrum, see Fig. 5. It can be observed that the spectrum of the excitation is approximately constant under $200 \mathrm{~Hz}$. Since all natural frequencies of interest of the arch fall within the range $30-200 \mathrm{~Hz}$ (as it will be shown next), it is possible to assert that the structure behaves as under white noise conditions over this frequency range. A preliminary numerical analysis [16] assisted in the decision making with regard to the sensors location (to avoid the placement of sensors in points where the modal curvatures could result null), the sampling frequency and the total measuring time. As far as the sensors are concerned, to record the dynamic response of the arch both accelerometers and strain gauges were used: the former were chosen for the level of accuracy and the latter for the direct estimation of the modal curvatures, parameters much more sensitive to damage than others. According to the configuration of the most significant numerical mode shapes, 11 measurement points evenly spaced along the arch were selected. These points were distributed along the two edges of the arch (front and back) for the accelerometers and along the two sides of the middle line of the arch (intrados and extrados) for the strain gauges, see Fig. 6. Such a distribution was considered good enough to obtain a fine resolution in terms of mode shapes. Note that only the in-plane response of the arch in normal and tangential direction was measured by the accelerometers since most of the modes numerically predicted had no significant modal displacements in the out-of-plane direction [9]. Altogether, 44 accelerations (22 in normal direction and 22 in 

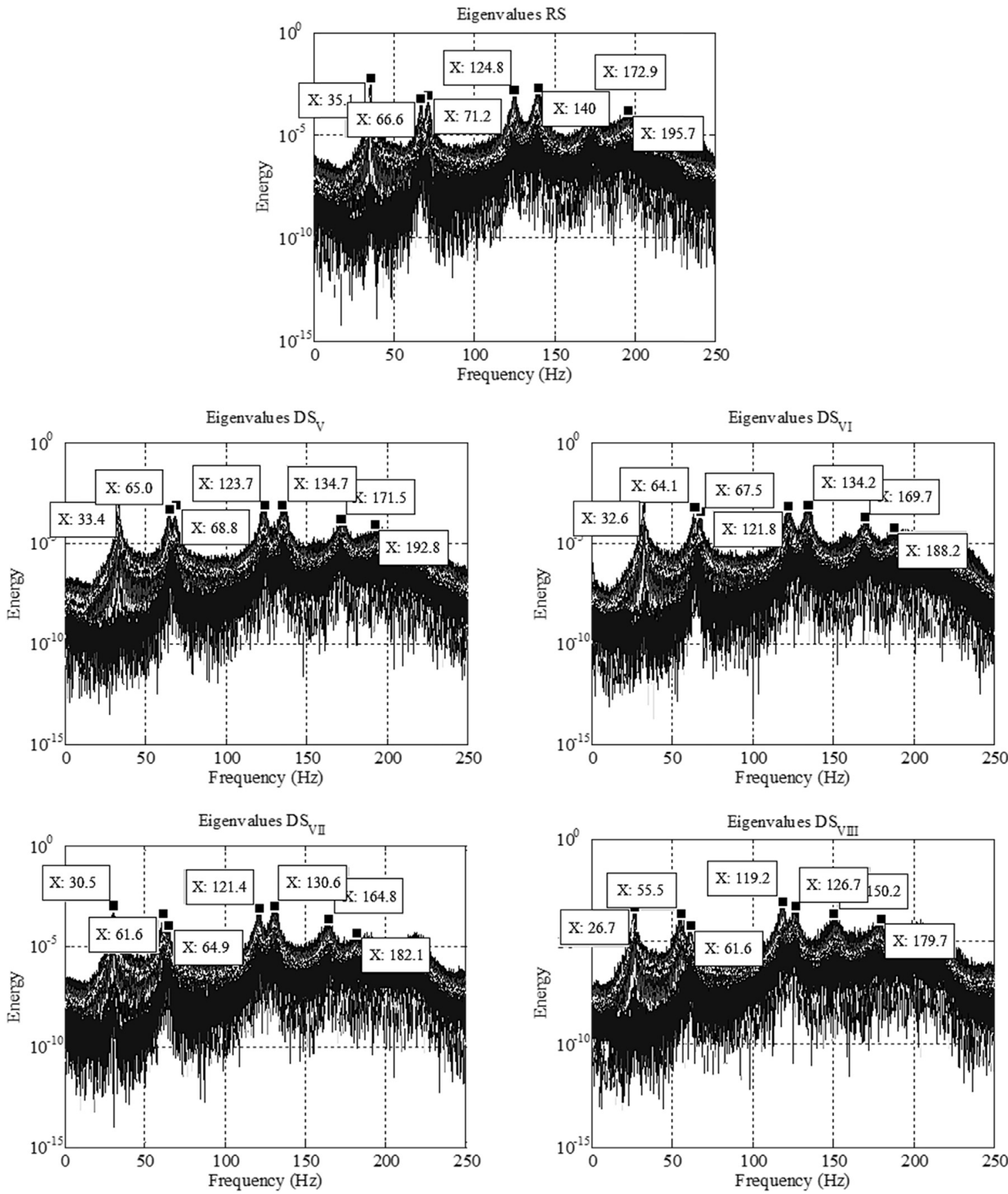

Fig. 8. Eigenvalues plotting for random impact excitation over the main DSs. The number of eigenvalues plotted equals the number of measurement points (22 acceleration responses).

tangential direction) and 22 strains (11 at the intrados and 11 at the extrados) were measured. A sampling frequency of $500 \mathrm{~Hz}$ and a minimum measuring time of $60 \mathrm{~s}$ were fixed, resulting in 30.000 data points per channel. The nodal response processes in terms of accelerations were acquired by ten setups, each one consisting of four reference sensors and four moving sensors. Regarding the strains measurements, only two setups with eight reference sensors (four at the intrados and four at the extrados) and eight or six moving sensors were used. Details about the test planning, the setups and the test equipment for the data acquisition are given in [16]. Table 1 presents the results obtained for the Reference Scenario (RS) in terms of mean frequencies values $(\omega)$ and damping ratios $(\xi)$. The standard deviation $(\sigma)$ and the Coefficient of Variation (CV) for both modal parameters are shown as well. The values were estimated by the Stochastic Subspace Identification (SSI) 
Table 2

Eigenfrequencies results: comparison between different identification techniques.

\begin{tabular}{|c|c|c|c|c|c|c|c|c|c|c|c|c|c|c|c|c|c|c|c|c|c|}
\hline \multirow[t]{2}{*}{ DS } & \multicolumn{3}{|l|}{ Mode 1} & \multicolumn{3}{|l|}{ Mode 2} & \multicolumn{3}{|l|}{ Mode 3} & \multicolumn{3}{|l|}{ Mode 4} & \multicolumn{3}{|l|}{ Model 5} & \multicolumn{3}{|l|}{ Model 6} & \multicolumn{3}{|l|}{ Model 7} \\
\hline & $f_{\mathrm{PSD}}(\mathrm{Hz})$ & $f_{\mathrm{SSI}}(\mathrm{Hz})$ & $\begin{array}{l}\Delta_{f} \\
(\%)\end{array}$ & $f_{\mathrm{PSD}}(\mathrm{Hz})$ & $f_{\text {SSI }}(\mathrm{Hz})$ & $\begin{array}{l}\Delta_{f} \\
(\%)\end{array}$ & $f_{\mathrm{PSD}}(\mathrm{Hz})$ & $f_{\mathrm{SSI}}(\mathrm{Hz})$ & $\begin{array}{l}\Delta_{f} \\
(\%)\end{array}$ & $f_{\mathrm{PSD}}(\mathrm{Hz})$ & $f_{\mathrm{SSI}}(\mathrm{Hz})$ & $\begin{array}{l}\Delta_{f} \\
(\%)\end{array}$ & $f_{\mathrm{PSD}}(\mathrm{Hz})$ & $f_{\text {SSI }}(\mathrm{Hz})$ & $\begin{array}{l}\Delta_{f} \\
(\%)\end{array}$ & $f_{\mathrm{PSD}}(\mathrm{Hz})$ & $f_{\mathrm{SSI}}(\mathrm{Hz})$ & $\begin{array}{l}\Delta_{f} \\
(\%)\end{array}$ & $f_{\mathrm{PSD}}(\mathrm{Hz})$ & $f_{\mathrm{SSI}}(\mathrm{Hz})$ & $\begin{array}{l}\Delta_{f} \\
(\%)\end{array}$ \\
\hline RS & 35.13 & 35.21 & -0.2 & 66.62 & 66.58 & +0.1 & 71.21 & 71.16 & +0.1 & 124.8 & 124.42 & +0.2 & 140.0 & 138.94 & +0.8 & 172.9 & 172.55 & +0.2 & 195.7 & 196.76 & -0.5 \\
\hline $\mathrm{DS}_{\mathrm{I}}$ & 35.11 & 35.17 & -0.2 & 66.77 & 66.67 & +0.2 & 71.08 & 71.22 & -0.2 & 125.0 & 124.55 & +0.4 & 139.1 & 138.93 & +0.1 & 173.4 & 173.41 & -0.0 & 196.4 & 196.13 & +0.1 \\
\hline $\mathrm{DS}_{\text {II }}$ & 34.99 & 35.17 & -0.5 & 66.06 & 66.72 & +0.5 & 71.44 & 71.25 & +0.3 & 126.2 & 124.64 & +1.3 & 138.9 & 138.99 & -0.1 & 174.5 & 173.30 & +0.7 & 195.9 & 196.83 & -0.5 \\
\hline $\mathrm{DS}_{\text {III }}$ & 35.02 & 35.11 & -0.3 & 66.79 & 66.73 & +0.1 & 70.77 & 71.14 & -0.5 & 125.7 & 124.65 & +0.8 & 140.1 & 138.86 & +0.9 & 173.7 & 173.12 & +0.3 & 196.3 & 196.71 & -0.2 \\
\hline $\mathrm{DS}_{\mathrm{IV}}$ & 35.17 & 34.83 & +1.0 & 66.31 & 66.45 & -0.2 & 71.38 & 70.76 & +0.9 & 125.6 & 124.49 & +0.9 & 138.4 & 138.33 & +0.1 & 172.8 & 172.88 & -0.1 & 197.8 & 196.30 & +0.8 \\
\hline $\mathrm{DS}_{\mathrm{V}}{ }^{*}$ & 33.34 & 33.20 & +0.4 & 65.03 & 64.62 & +0.6 & 68.83 & 68.33 & +0.7 & 123.7 & 123.10 & +0.5 & 134.7 & 135.61 & -0.7 & 171.5 & 171.10 & +0.2 & 192.8 & 193.43 & -0.3 \\
\hline $\mathrm{DS}_{\mathrm{VI}}$ & 32.56 & 32.42 & +0.4 & 64.07 & 63.84 & +0.4 & 67.54 & 67.18 & +0.5 & 121.8 & 122.14 & -0.3 & 134.2 & 134.31 & -0.1 & 169.7 & 169.54 & +0.1 & 188.2 & 186.10 & +1.1 \\
\hline $\mathrm{DS}_{\mathrm{VII}}$ & 30.53 & 30.17 & +1.2 & 61.61 & 61.29 & +0.5 & 64.90 & 64.06 & +1.3 & 121.4 & 120.26 & +0.9 & 130.6 & 130.71 & -0.1 & 164.8 & 163.93 & +0.5 & 182.1 & 182.37 & -0.2 \\
\hline $\mathrm{DS}_{\mathrm{VIII}}$ & 26.66 & 26.13 & +2.0 & 55.53 & 55.28 & +0.5 & 61.33 & 61.33 & +0.5 & 119.2 & 117.59 & +1.4 & 126.7 & 125.95 & +0.6 & 150.2 & 151.43 & -0.8 & 179.7 & 176.33 & +1.9 \\
\hline |Avrg| & - & - & +0.7 & - & - & +0.3 & - & - & +0.6 & - & - & +0.7 & - & - & +0.4 & - & - & +0.3 & - & - & +0.6 \\
\hline
\end{tabular}

* Damage scenario in which the first crack was detected during the static tests. 
Table 3

Eigenfrequencies decrease over the eight damage scenarios.

\begin{tabular}{|c|c|c|c|c|c|c|c|}
\hline \multirow[t]{2}{*}{ Damage scenario } & \multicolumn{7}{|c|}{ Eigenfrequencies decrease $(\mathrm{Hz})$} \\
\hline & Mode 1 & Mode 2 & Mode 3 & Mode 4 & Mode 5 & Mode 6 & Mode 7 \\
\hline RS & - & - & - & - & - & - & - \\
\hline $\mathrm{DS}_{\mathrm{I}}$ & -0.02 & +0.15 & -0.13 & +0.20 & -0.90 & +0.50 & +0.70 \\
\hline $\mathrm{DS}_{\mathrm{II}}$ & -0.14 & +0.44 & +0.23 & +1.40 & -1.10 & +1.60 & +0.20 \\
\hline $\mathrm{DS}_{\text {III }}$ & -0.11 & +0.17 & -0.44 & +0.90 & +0.10 & +0.80 & +0.60 \\
\hline $\mathrm{DS}_{\mathrm{IV}}$ & +0.04 & -0.31 & +0.17 & +0.80 & -1.60 & -0.10 & +2.10 \\
\hline $\mathrm{DS}_{\mathrm{V}}$ & -1.79 & -1.59 & -2.38 & -1.10 & -5.30 & -1.40 & -2.90 \\
\hline $\mathrm{DS}_{\mathrm{VI}}$ & -2.57 & -2.55 & -3.67 & -3.00 & -5.80 & -3.20 & -7.50 \\
\hline $\mathrm{DS}_{\mathrm{VII}}$ & -4.60 & -5.01 & -6.31 & -3.40 & -9.40 & -8.10 & -13.60 \\
\hline $\mathrm{DS}_{\mathrm{VIII}}$ & -8.47 & -11.09 & -9.58 & -5.60 & -13.30 & -22.70 & -16.00 \\
\hline$\Delta_{f}(\%)$ & -24.1 & -16.6 & -13.5 & -4.5 & -9.5 & -13.1 & -8.2 \\
\hline
\end{tabular}

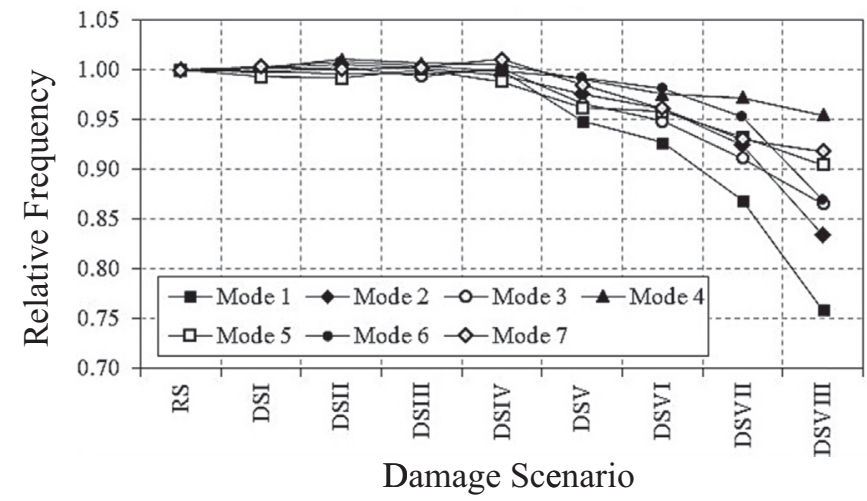

Fig. 9. Relative frequency values for the identified modes along the dynamic tests.

technique implemented in ARTeMIS (SVS, 2006). The modes of vibration for the RS (undamaged condition) are shown in Fig. 7.

\subsubsection{Experimental damage analysis}

The damage analysis over the eight DSs experimentally induced was performed through non-model based methods. This approach consists in analysing the damage by studying direct changes in the modal parameters of the arch as well as changes in the derivatives of these modal parameters. A group of Vibration-Based Damage Identification Methods (VBDIMs) was selected for this purpose. This approach will be discussed in the next sections together with the results obtained by the spectrum-driven damage identification method.

\section{Spectral damage analysis of the arch}

After computing the second order moments of the nodal response processes, three square PSD matrices were built for each scenario: two $[22 \times 22]$ matrices from acceleration responses in both normal $(z)$ and tangential $(x)$ direction and a $[11 \times 11]$ matrix from strain measurements. In order to identify the modal parameters and proceed with the damage analysis, each matrix was then decomposed in eigenvalues and eigenvectors according to what described in Section 2.1.

\subsection{Eigenvalues analysis}

The eigenvalues decomposition, first performed with regard to the RS and then applied to the eight DSs, allowed to identify the eigenfrequencies to use as qualitative indicators in the damage analysis with global parameters. This type of analysis is essentially based on the observation of shifts in the resonant frequency values. If accurately estimated, direct changes in these modal parameters are proved to be a sensitive indicator of global structural damage. Fig. 8 shows the eigenvalues plotting corresponding to the selected scenarios. Each eigenvalue features the spectral distribution of the average energy of each vibration mode. As it can be seen, seven modes are clearly identified in every single stage. The results for the nine consecutive scenarios using the PSD technique are presented in Table 2 together with the ones obtained from the SSI technique [19] during the experimental campaign (see also Table 1). The low values of percentage error indicate a good agreement among the frequencies identified by the two tools. Both techniques also detected small increases in the 


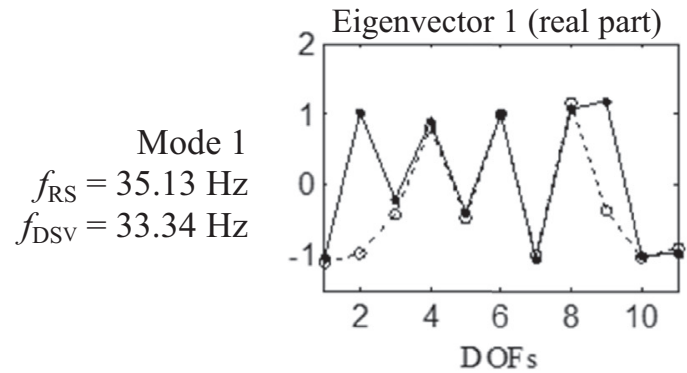

Eigenvector 2 (real part)

Mode 2 $f_{\mathrm{RS}}=66.62 \mathrm{~Hz}$ $f_{\mathrm{DSV}}=65.03 \mathrm{~Hz}$

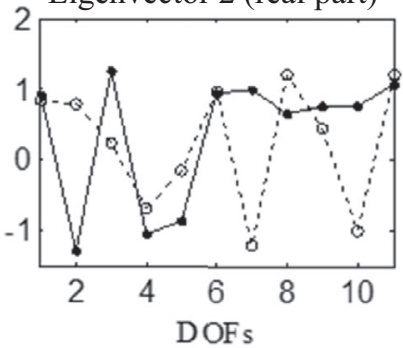

Eigenvector 3 (real part)

Mode 3

$$
f_{\mathrm{RS}}=71.21 \mathrm{~Hz}
$$

$f_{\mathrm{DSV}}=68.83 \mathrm{~Hz}$

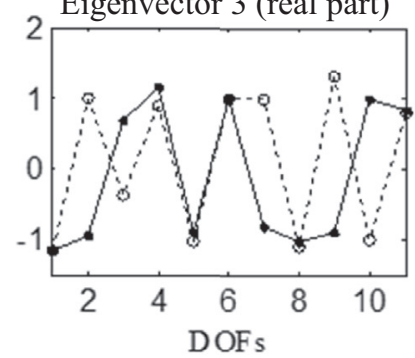

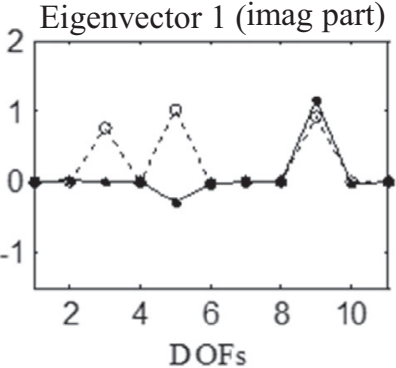

Eigenvector 2 (imag part)

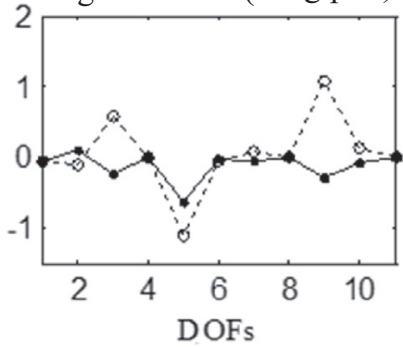

Eigenvector 3 (imag part)

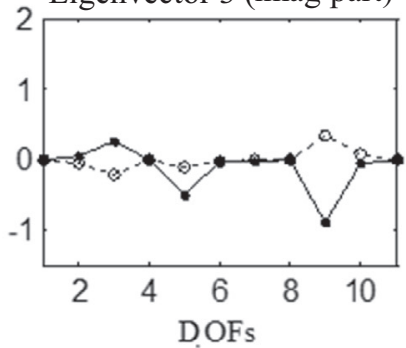

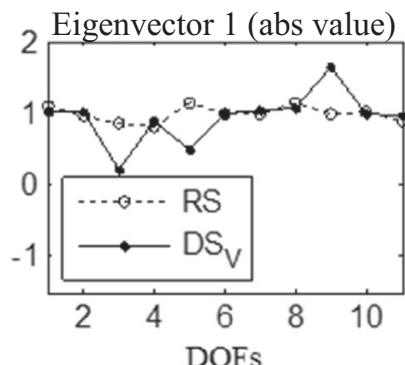

Eigenvector 2 (abs value)

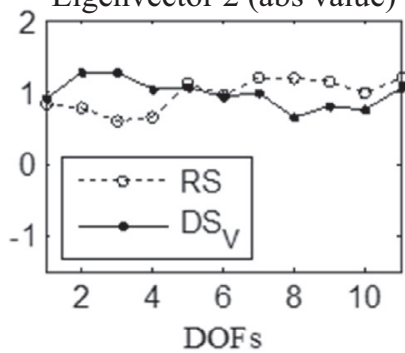

Eigenvector 3 (abs value)

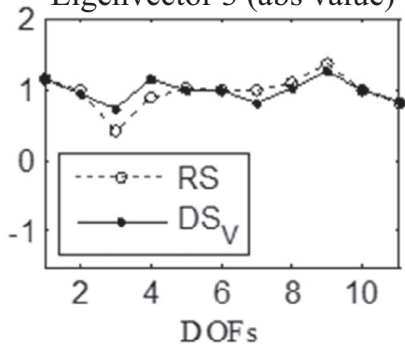

Fig. 10. Eigenvectors plotting for strain data: comparison between real parts (left), imaginary parts (centre) and absolute values (right) of the first three

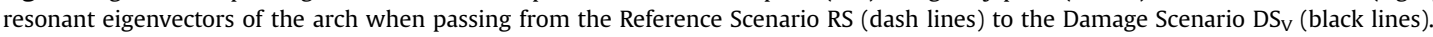

frequency values before the occurrence of the first crack. In fact, it is observed that during the initial damage scenarios the masonry arch is hardening, resulting in a stiffness increase near the occurrence of the first crack (Fig. 3). With the applied load/unload and the consequent cracking process, the initial stored energy is progressively dissipated during vibrating motions and the structural stiffness of the arch degrades with the increase of damage. As expected, this led to considerable changes in terms of eigenfrequencies, recording decreases up to $24.1 \%$ and $16.6 \%$ for the first two modes (see Table 3 ). According to the experimental evidence, the first significant frequency drop is observed in $\mathrm{DS}_{\mathrm{V}}$ and it mostly concerns the lower modes (see Fig. 9).

\subsection{Eigenvectors analysis}

In order to enhance the damage investigation procedure and to move from a global to a local damage identification, complex eigenvectors belonging to different scenarios are plotted and analysed. As described in Section 3.2.1, four cracks progressively appeared during the static tests: crack $c_{1}$ at the intrados between positions $\mathrm{P} 3$ and P4; crack $c_{2}$ at the right support of the arch, i.e. position P11; crack $c_{3}$ at the extrados between positions P8 and P9; and crack $c_{4}$ at the left support of the arch, i.e. position P1. Since the only crack visually detected during the experimental campaign was the one occurring between positions $\mathrm{P} 3$ and $\mathrm{P} 4$ in $\mathrm{DS}_{\mathrm{V}}$ ( $\operatorname{crack} c_{1}$ ), the eigenvectors analysis has focused on the comparison of $\mathrm{DS}_{\mathrm{V}}$ first with the $\mathrm{RS}$, and then with the previous scenario $\mathrm{DS}_{\mathrm{IV}}$. Nodes 3 and 4 are taken as initial target points to weight the accuracy and reliability of the information provided by the eigenvectors in terms of damage location when comparing their imaginary part rather than their real counterpart. The aim is to understand indeed which part is the most relevant for damage identification. With this purpose, real and imaginary parts of each resonant eigenvector are plotted, whether separately or jointly (through the absolute value of their module), and compared. Fig. 10 shows this comparison for the first three resonant eigenvectors of the arch when passing from Reference Scenario (RS) to Damage Scenario V (DS $)$. Analogously, Fig. 11 displays the same comparison but with reference to two consecutive DSs, namely $\mathrm{DS}_{\mathrm{IV}}$ and $\mathrm{DS}_{\mathrm{V}}$. Unlike the inconclusive comparison between the real parts, the comparison between the imaginary parts of each eigenvector alerts the attention on nodes 3, 5 and 9 as possible damage locations. The comparison between absolute values also catches nodes close to the real 


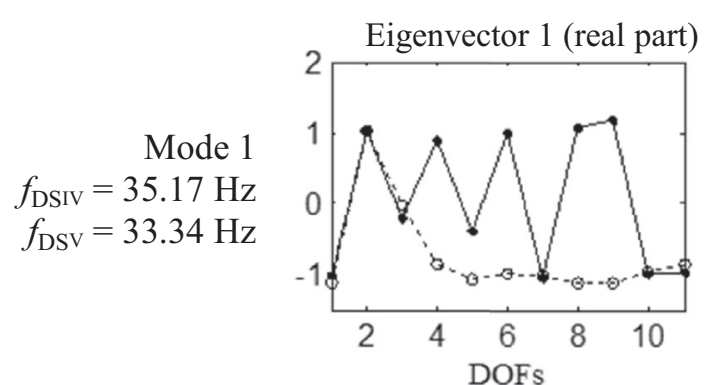

Eigenvector 2 (real part)

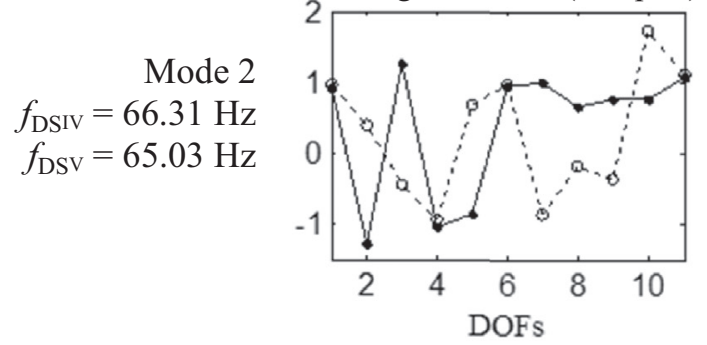

Eigenvector 3 (real part)

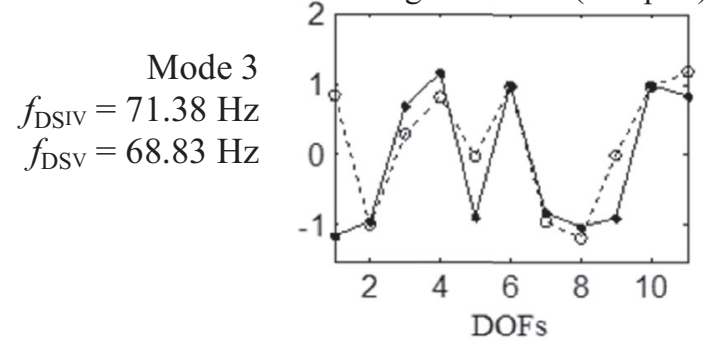

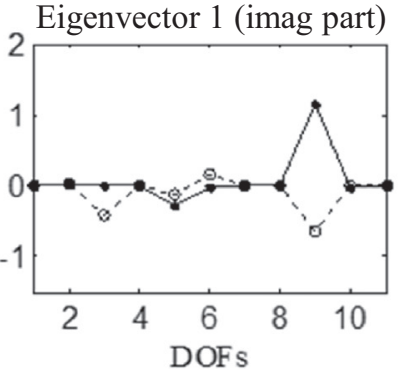

Eigenvector 2 (imag part)

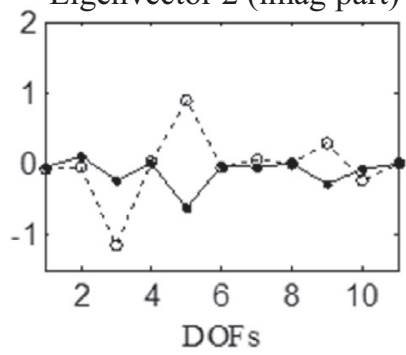

Eigenvector 3 (imag part)

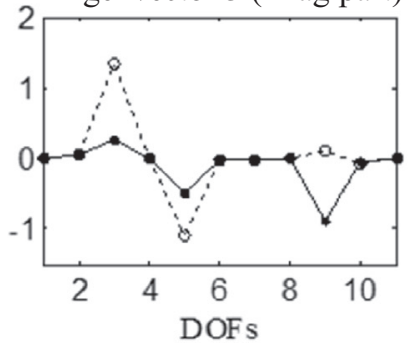

Eigenvector 1 (abs value)

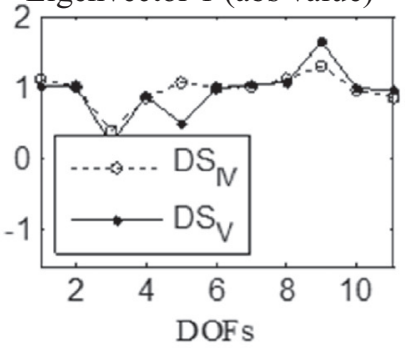

Eigenvector 2 (abs value)

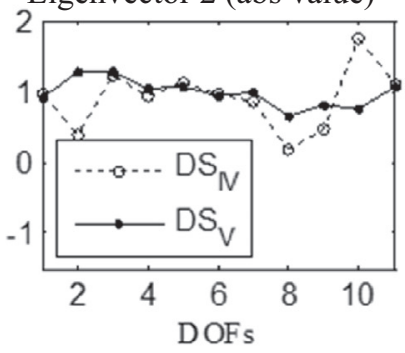

Eigenvector 3 (abs value)

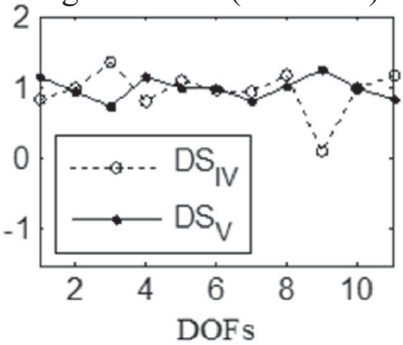

Fig. 11. Eigenvectors plotting for strain data: comparison between real parts (left), imaginary parts (centre) and absolute values (right) of the first three resonant eigenvectors of the arch when passing from Damage Scenario $\mathrm{DS}_{\mathrm{IV}}$ (dash lines) to Damage Scenario DS $($ black lines).

cracks. This behaviour conceivably originates from the Hermitian nature of the PSD matrix. As such, this matrix consists of a real part (co-spectrum) and an imaginary part (quad-spectrum). The former is related to the in-phase motion of the structure, whereas the latter is related to the out-of-phase components of the structural response. As structures are more prone to experience local damage in case of out-of-phase vibrations due to the unsynchronised movements of the different structural nodes, it follows that phase differences in the system's response may act as a damage indicator. This mechanical phenomenon explains why the imaginary part of the PSD matrix provides more information to locate damage with respect to its real counterpart.

Regarding the eigenvectors analysis for acceleration responses (Figs. 12 and 13), it can be seen that neither real nor imaginary part of the compared resonant eigenvectors gives reliable warnings about the damage position. On the other hand, the comparison between absolute values pinpoints the target nodes P3 and P4 or the ones close by and draws the attention around position P8/P9 as well. Weighing the contribution of both real and imaginary part is likely the best approach. However, given the difficulty of the subject, more investigation is needed to take this issue forward, also involving new experimental results.

\section{Discussion of the results}

Although changes in the complex eigenvectors can be used as spatial indicators in the damage analysis, they can yield false alarms if their respective eigenvalues are left out of consideration. Briefly, even if three nodes are caught as possible damage locations when comparing single eigenvectors belonging to different DSs, this does not mean the three nodes are all and evenly damaged. In fact, each eigenvector is characterised by its own energy of vibration, thus the contribution of all eigenvectors appropriately weighed through their respective eigenvalues should account for the damage analysis. Hence the combination of both eigenparameters for the estimation of spectral modes and the computation of the spectral damage index, see Eq. (10).

As modal curvatures are more sensitive to damage than other parameters, the algorithm is first adopted by using the spectral parameters obtained from strain measurements. Afterward, the procedure is repeated for the spectral quantities 


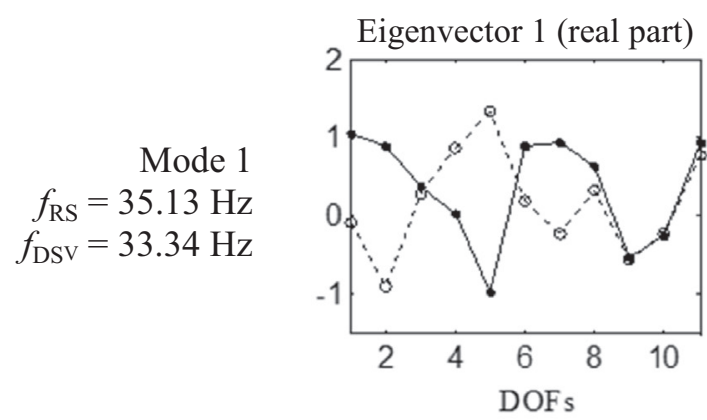

Eigenvector 2 (real part)

Mode 2

$f_{\mathrm{RS}}=66.62 \mathrm{~Hz}$

$f_{\mathrm{DSV}}=65.03 \mathrm{~Hz}$

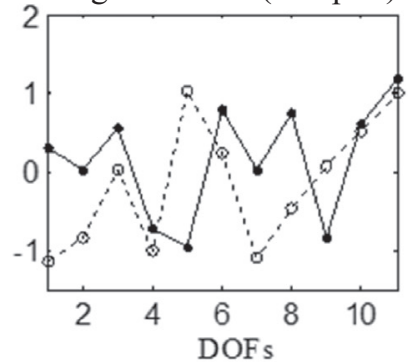

Eigenvector 3 (real part)

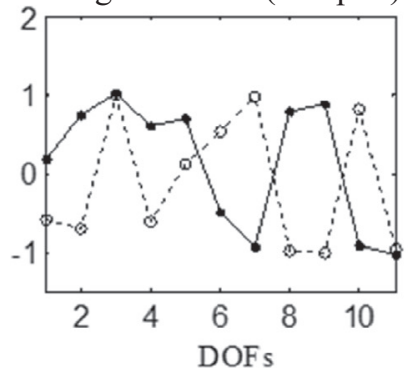

Eigenvector 1 (imag part)

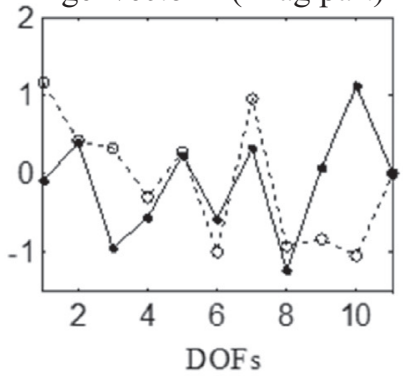

Eigenvector 2 (imag part)

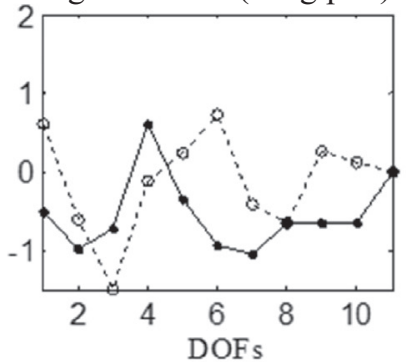

Eigenvector 3 (imag part)

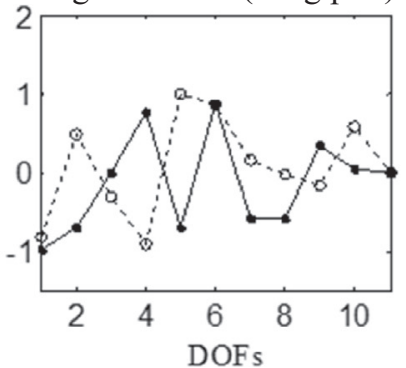

Eigenvector 1 (abs value)

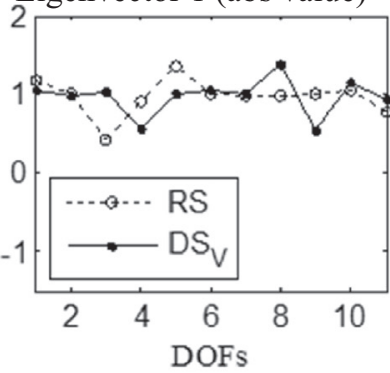

Eigenvector 2 (abs value)

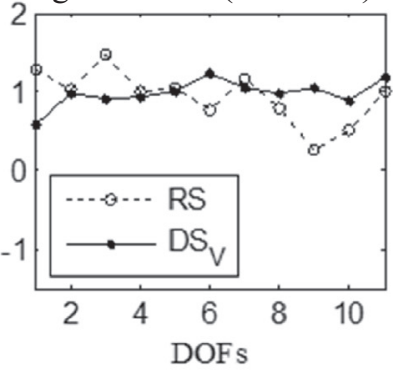

Eigenvector 3 (abs value)

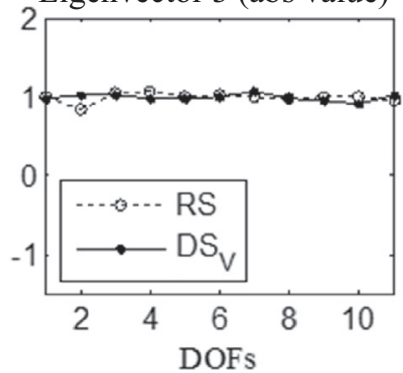

Fig. 12. Eigenvectors plotting for acceleration responses along the back edge: comparison between real parts (left), imaginary parts (centre) and absolute values (right) of the first three resonant eigenvectors of the arch when passing from Reference Scenario RS (dash lines) to Damage Scenario DS $\mathrm{V}_{\mathrm{V}}$ (black lines).

estimated from acceleration responses. Besides the comparison with the RS, each DS is progressively compared with the previous one, so as to follow the damage evolution along the testing. In order to study the sensitivity of the algorithm to the frequency bandwidth considered, two types of damage index are computed and compared: a Broad-Band (BB) index and a Narrow-Band (NB) index. As detailed in Section 2.2, the former is based on the difference between spectral modes weighed over the whole frequency domain by the respective non-zero singular values (Eq. 10); the latter relies on the difference between resonant spectral modes, each one weighed by its relevant eigenvalue at the resonant frequency (Eq. 11). The first damage scenarios are not taken into account in the comparison for the following reasons: (i) the first crack notoriously appeared in $\mathrm{DS}_{\mathrm{V}}$, (ii) the measurements of the initial scenarios resulted to be affected by the adjustments of the structure with the applied load. Note that the number of bars in the index plotting coincides with the measured DOFs, whereas the size of each bar gives a rough estimate of the size of damage affecting that particular node or area close by.

\subsection{Comparison with the experimental damage analysis}

\subsubsection{Strain data}

The results of the spectral-based damage localisation analysis for strain measurements are presented in Fig. 14. As it can be observed, in the comparisons with the RS, the NB index pinpoints the damage exactly in the vicinity of the four nodes in which the actual cracks occurred, namely at positions P1, P4, P8 and P11. With the exception of node P1 (left support), the other three nodes are all highlighted as possible damage locations from $\mathrm{DS}_{\mathrm{V}}$. In the relative comparisons with the previous configurations, the NB index identifies again the damage nearby the actual damaged nodes (P1, P3, P9 and P11), but according to a different crack sequence. The first two cracks appear at positions P3 and P9 between DS $S_{I V}$ and DS $S_{V}, w_{\text {ereas }}$ the cracks at positions P1 and P11 occur between $\mathrm{DS}_{\mathrm{V}}$ and $\mathrm{DS}$ VI. In the last phase, a slight peak at position P7 seems to detect a possible new crack. Although no crack was visually caught in this measuring point during the experimental tests, it is possible that some micro-crack appeared due to the stress redistribution. 
Eigenvector 1 (real part)

Mode 1

$f_{\text {DSIV }}=35.17 \mathrm{~Hz}$

$f_{\mathrm{DSV}}=33.34 \mathrm{~Hz}$

Mode 2

$f_{\mathrm{DSIV}}=66.31 \mathrm{~Hz}$

$f_{\text {DSV }}=65.03 \mathrm{~Hz}$

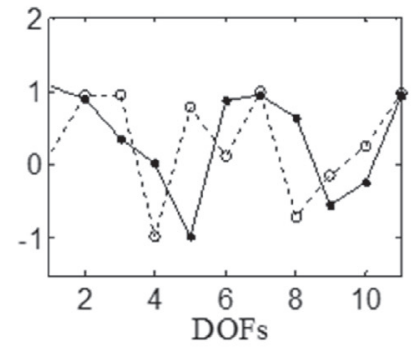

Eigenvector 2 (real part)

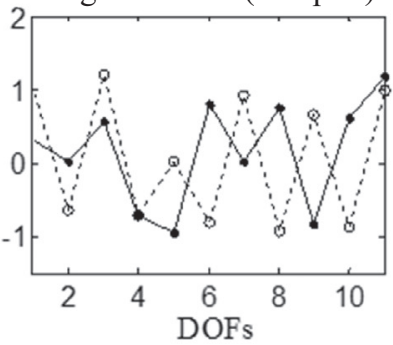

Eigenvector 3 (real part)

Mode 3

$f_{\text {DSIV }}=71.38 \mathrm{~Hz}$

$f_{\mathrm{DSV}}=68.83 \mathrm{~Hz}$

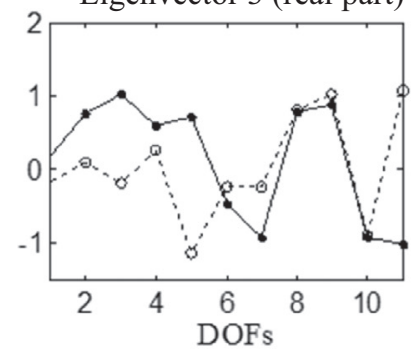

Eigenvector 1 (imag part)

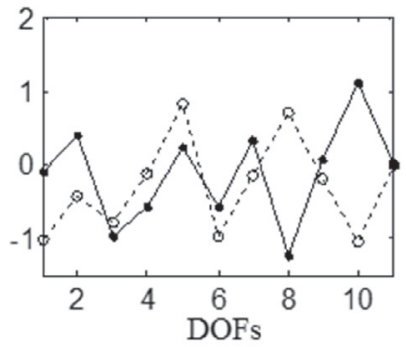

Eigenvector 2 (imag part)

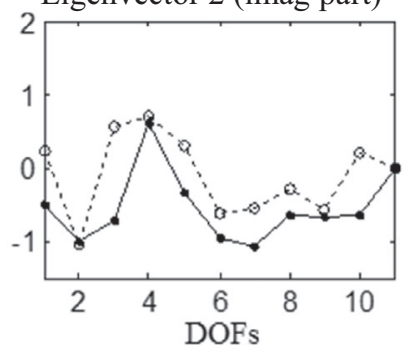

Eigenvector 3 (imag part)

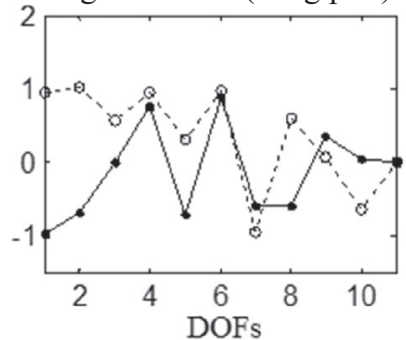

Eigenvector 1 (abs value)

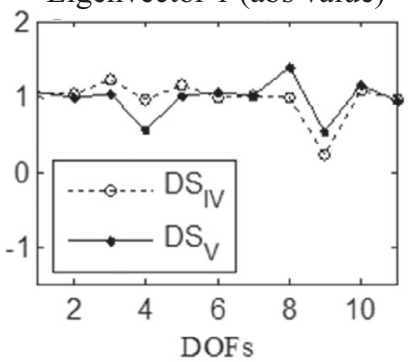

Eigenvector 2 (abs value)

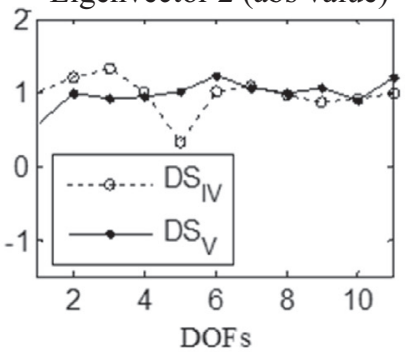

Eigenvector 3 (abs value)

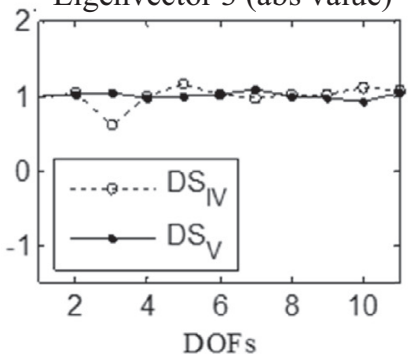

Fig. 13. Eigenvectors plotting for acceleration responses along the back edge: comparison between real parts (left), imaginary parts (centre) and absolute values (right) of the first three resonant eigenvectors of the arch when passing from Damage Scenario DS $\mathrm{IV}_{\mathrm{IV}}$ (dash lines) to Damage Scenario DS $\mathrm{V}$ (black lines).

Regarding the comparisons with the RS for the BB index, all the target nodes are caught: besides P1 and P11 in DS $\mathrm{V}$ and $\mathrm{P} 3$ and P9 in $\mathrm{DS}_{\mathrm{VI}}$, also peaks at positions P4 and P8 are exhibited in the last two scenarios. As far as the relative comparisons are concerned, the BB index detects again the presence of damage in the nodes nearby the observed experimental cracks, namely P1, P4, P8 and P11. Nevertheless, unexpected peaks at positions P6 and P7 show up between DS and DS $S_{\mathrm{VI}}$.

Even though catching a sole crack sequence was not possible, the overall results of the spectral damage analysis for strain measurements are rather consistent: the damage is successfully detected and the cracks are localised at a very early stage. In addition, narrowing the frequency bandwidth of interest in the damage index computation helps to reduce the influence of noise in the data: in fact, unlike the BB index, the NB index exhibits clear-cut peaks and a crack sequence closer to the experimental evidence, especially in the relative comparison.

\subsubsection{Acceleration data}

In regard to the spectral damage analysis for acceleration responses, it is noted that only the spectral output signals in normal direction have been used for the index computation. The spectral output signals in tangential direction displayed a low physical magnitude, so their contribution in the damage analysis could be neglected. The results are presented in Figs. 15 and 16. The comparison with the RS exhibits consistent results between front and back edges. In detail, according to the BB damage index, if the contribution of both edges is considered, the predicted cracks have the following sequence: P3,

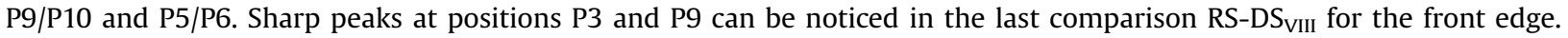
Concerning the NB damage index, although minor peaks appear at positions P3, P5 and P7, only position P9 is clearly indicated as possible damage location for both front and back edge.

For the case of progressive comparisons between consecutive DSs, the BB index shows quite confusing peaks: for instance, when comparing $\mathrm{DS}_{\mathrm{IV}}$ with $\mathrm{DS}$, no peak is clearly detected; whereas cracks at positions P2/P3, P4, P7, P9 and P10 are pinpointed when proceeding with the comparisons. Still, unexpected peaks at positions P5/P6 are shown. On the contrary, for the case of NB index, the damage analysis gives much more consistent and reliable results. The target nodes at positions P3/P4 and P8/P9 are well caught, but additional nodes at positions P2 and P6/P7 are also identified as possible damage locations. 
RS-DS $\mathrm{V}$
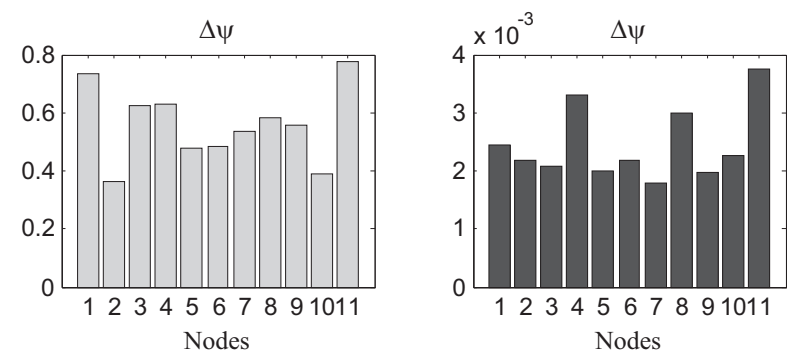

RS-DS ${ }_{\mathrm{VI}}$
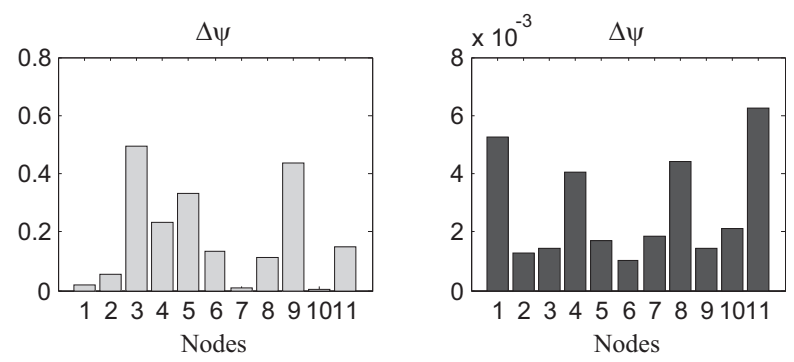

RS-DS
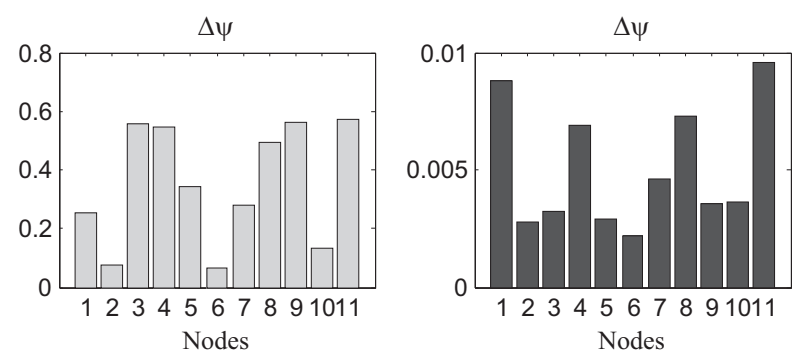

RS-DS
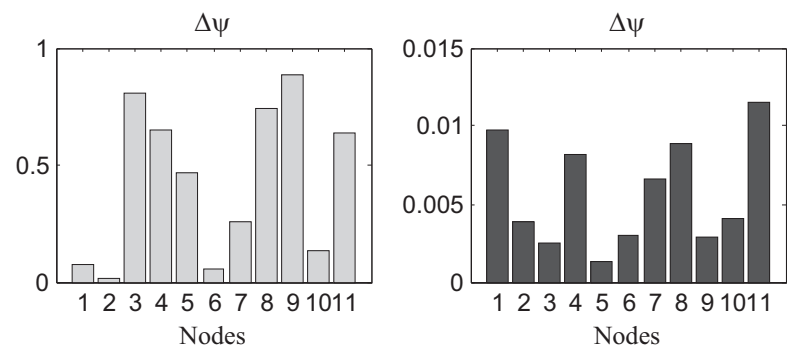

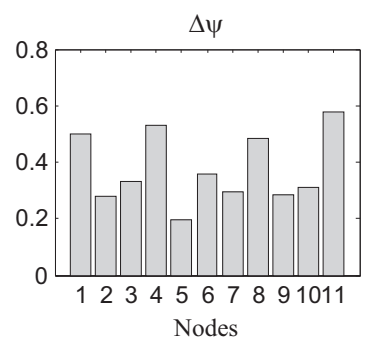

$\mathrm{DS}_{\mathrm{IV}}-\mathrm{DS}_{\mathrm{V}}$

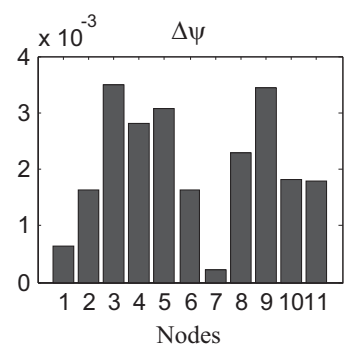

$\mathrm{DS}_{\mathrm{V}}-\mathrm{DS}_{\mathrm{VI}}$
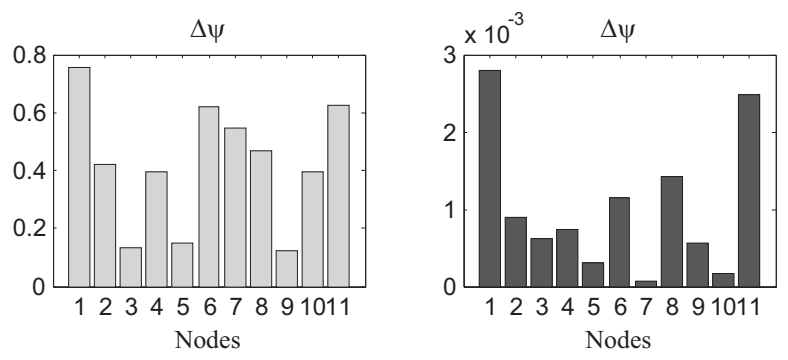

$\mathrm{DS}_{\mathrm{VI}}-\mathrm{DS}_{\mathrm{VII}}$
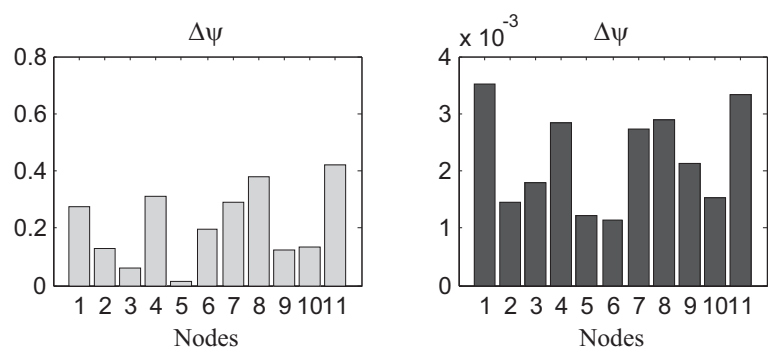

$\mathrm{DS}_{\mathrm{VII}}-\mathrm{DS}_{\mathrm{VIII}}$
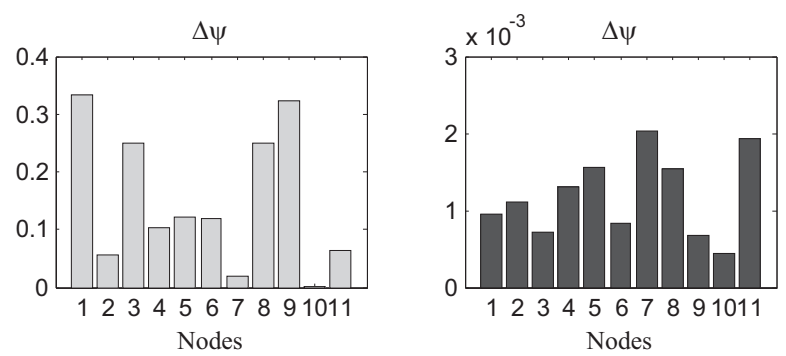

Fig. 14. Spectral damage analysis results for strain measurements by BB index (light grey bars) and NB index (dark grey bars): comparison with the RS and progressive comparison between DSs. Here, BB indicates Broad-Band and NB indicates Narrow-Band.

Altogether, it can be stressed that the spectral damage analysis from acceleration responses shows more accurate results for the BB index when performing the comparison with the RS, whereas more reliable results are obtained from the NB index in case of relative comparisons with the previous configurations.

\subsubsection{Final remarks}

Considering all the above observations, it is possible to conclude that the spectrum-driven damage identification based on data from strain measurements allowed to achieve better results with respect to the analysis carried out making use of acceleration responses. This result was somehow predictable. In fact, it is known that modal curvatures (and so modal strains) are more sensitive to structural changes than other modal parameters, especially when dealing with localised phenomena such as structural damage. This explains why strain gauges are often used in dynamic tests aimed at damage 


\section{Front Edge}
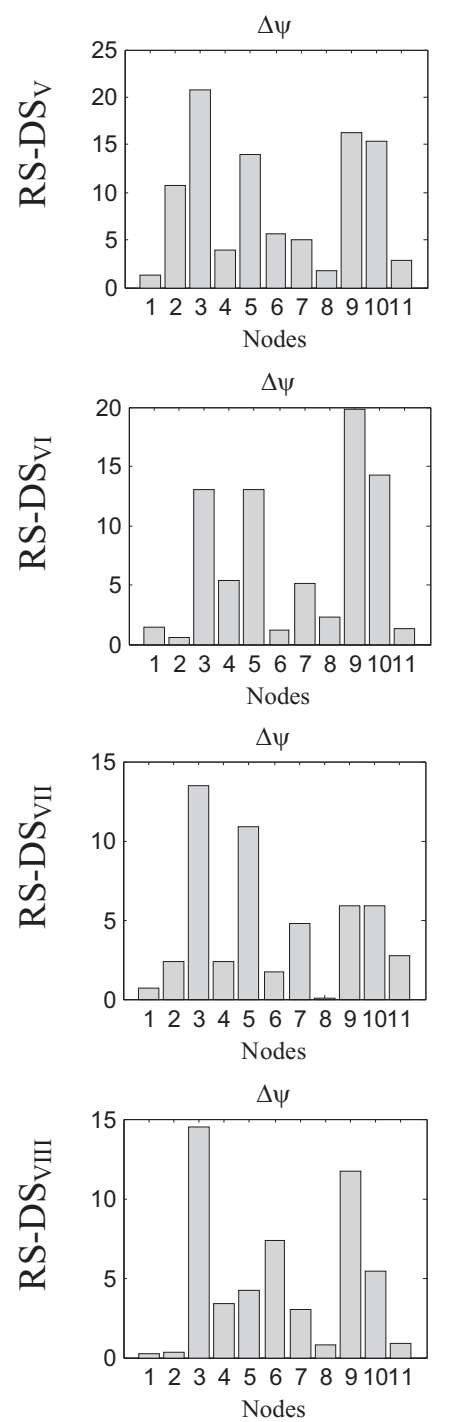
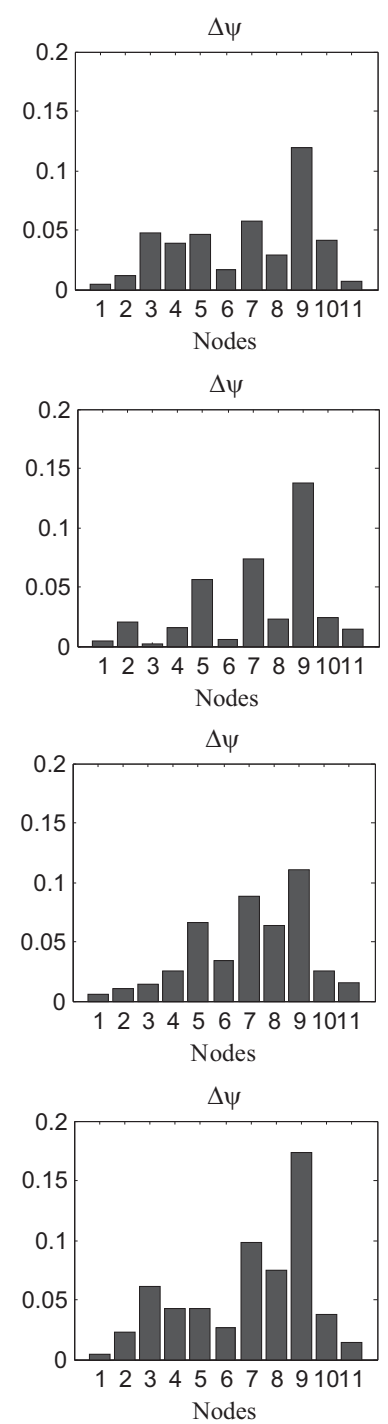

Back Edge
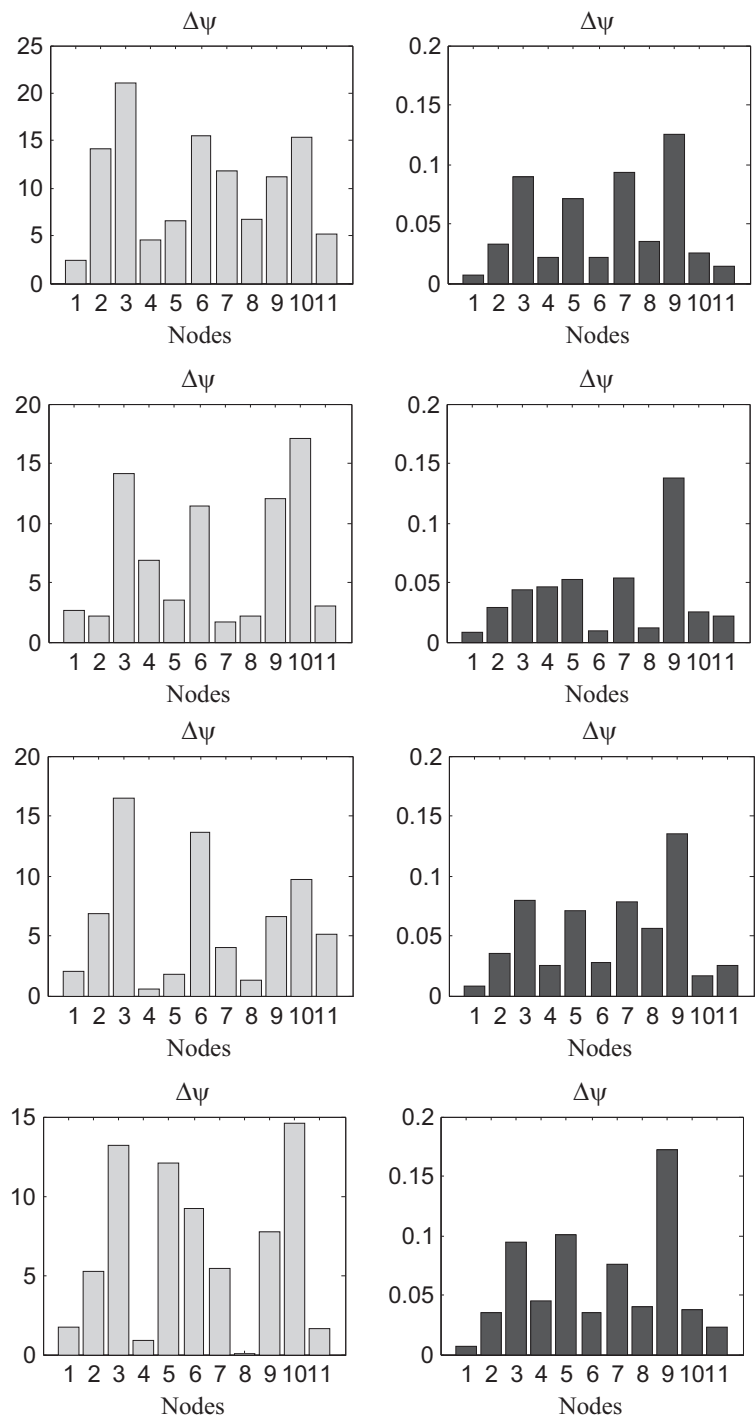

Fig. 15. Spectral damage analysis results for acceleration responses by BB index (light grey bars) and NB index (dark grey bars): comparison with the RS for front and back edge.

identification. Although accelerometers are necessary to ensure a good level of accuracy for the dynamic response, strain gauges are needed for the direct estimation of modal curvatures in order to have more information about the damage position. It is worth noting that modal curvatures are valuable quantities for damage identification analysis under the assumption of Euler-Bernoulli hypotheses: the bending stiffness and normal stiffness are directly related to the curvatures and the average strains in the structure, respectively. Therefore, both modal curvatures and strains will conceivably result more affected than acceleration responses by the loss of stiffness due to damage occurrence. Yet, since damage might yield the same modal curvatures changes at different measurement points, the spectral damage analysis does not rely directly on the modal curvatures themselves but exploits the second order properties of these modal parameters to identify the damage. This reveals the value-added of upgraded spectral analysis methods for damage localisation purposes. Finally, it is stressed that the NB damage index proved to be more accurate and reliable than the BB index in most of the cases, especially with regard to the crack sequence.

\subsection{Comparison with other VBDIMs}

In order to have an additional criterion to weigh and evaluate the performance of the spectral method, the analytical results have also been compared with the ones obtained from other VBDIMs, i.e. the Co-ordinate Modal Assurance Criterion (COMAC), the Parameter Method (PM), the Mode Shape Curvature Method (MSCM), the Sum of all Curvature Errors method 


\section{Front Edge}
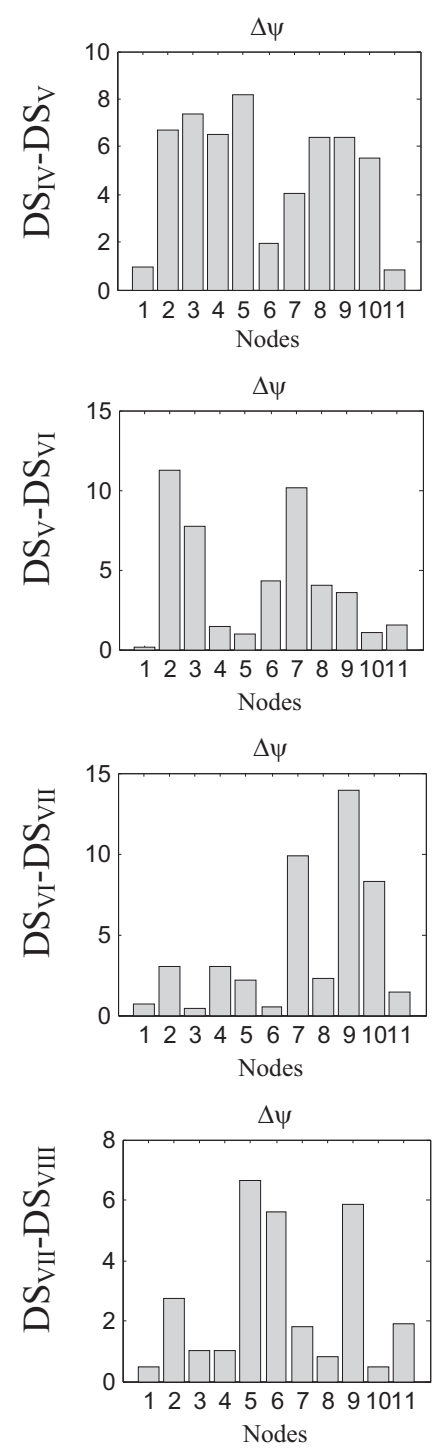
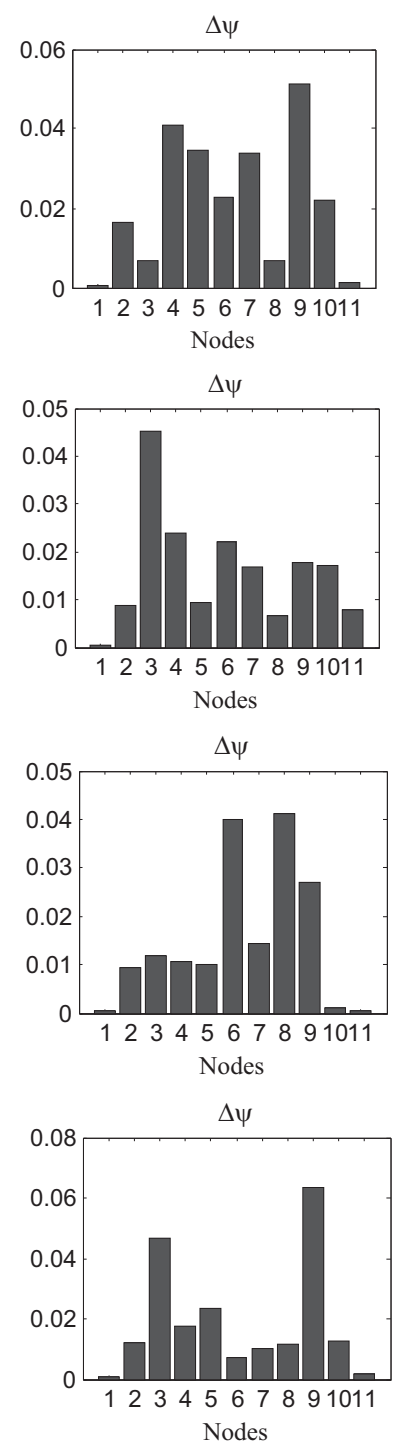

\section{Back Edge}
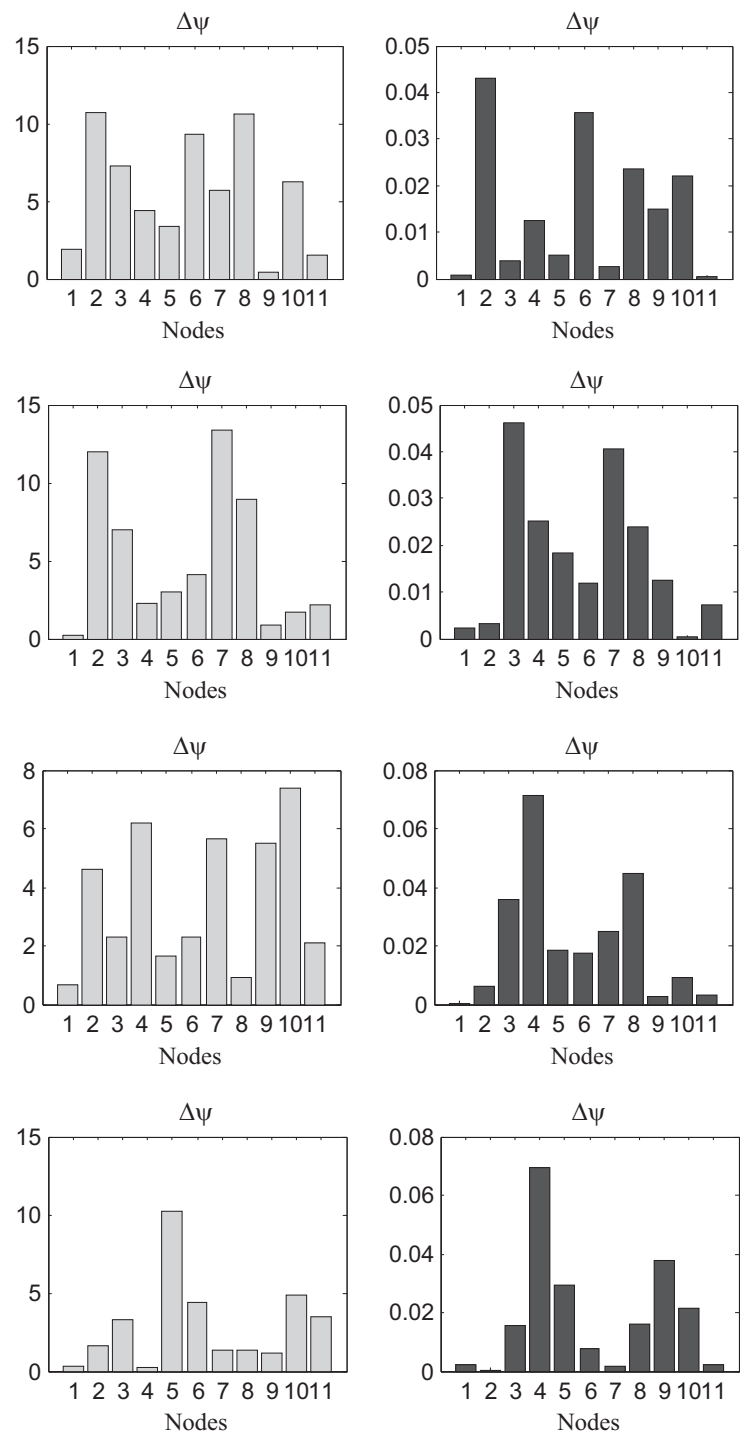

Fig. 16. Spectral damage analysis results for acceleration responses by BB index (light grey bars) and NB index (dark grey bars): relative comparison between DSs for front and back edge.

(SCE), the Damage Index Method (DIM), Changes in Flexibility Matrix method (CFM). The damage indexes used by each technique are briefly listed in Table 4. Mode shapes or modal curvatures are the parameters required for their computation. A complete and exhaustive description of these methods and the damage analysis carried out is given in [16]. In this paper, only the results from measured curvatures are reported since the ones obtained from modal displacements were mostly inconclusive or insensitive to damage.

Fig. 17 shows the results of the main steps of the damage investigation so as to have a quick survey on the performance of the different methods and to make the comparison with the spectral approach easier. The analysis focused on two damage scenarios: the damage scenario corresponding to the occurrence of the first crack $\left(\mathrm{DS}_{\mathrm{V}}\right)$ and the damage scenario in which all cracks became visible, viz. the last one ( $\left.\mathrm{DS}_{\mathrm{VIII}}\right)$. The spectral approach was applied to assess the changes in the aforementioned damage scenarios with respect to the initial undamaged state (RS) and to the prior state (DS $\mathrm{IV}_{\mathrm{IV}}$ or $\mathrm{DS}_{\mathrm{VII}}$ ). Regarding the results of the comparison between RS and $\mathrm{DS}_{\mathrm{V}}$, it can be seen that MSCM, PM and CFM univocally detected the occurrence of cracks at positions P1 and P11 (left and right support), DIM and SCE showed only one peak at position P1, whereas the COMAC resulted inconclusive. The notorious first crack was not detected at this stage, but in prior comparisons. Analysing the results from the relative comparison between $\mathrm{DS}_{\mathrm{IV}}$ and $\mathrm{DS}_{\mathrm{V}}$, it emerged that the COMAC was again inconclusive or insensitive to damage, MSCM, PM and CFM exhibited similar peaks converging towards positions P1 and P11, the SCE identified an additional damage location at position P7 besides the ones at the supports and the DIM pinpointed the 
Table 4

List of selected non-model based damage identification methods.

\begin{tabular}{|c|c|c|}
\hline Method & Modal Parameters & Damage Index \\
\hline COMAC & $\begin{array}{l}\text { Mode shapes } \phi \text { or } \\
\text { modal curvatures } \phi^{\prime \prime}\end{array}$ & $\left|\sum_{j=1}^{m} \varphi_{i, j}^{u_{i} d_{i, j}^{d}}\right|^{2}$ \\
\hline & & $\overline{\sum_{j=1}^{m}\left(\varphi_{i, j}^{u}\right)^{2} \sum_{j=1}^{m}\left(\varphi_{i, j}^{d}\right)^{2}}$ \\
\hline PM & Mode shapes $\phi$ or & \\
\hline & Modal curvatures $\phi^{\prime \prime}$ & $\sum_{j=1}^{m} \mid \varphi_{j}^{d} \frac{\omega_{j}^{j}}{\omega_{j}^{d}}-\varphi_{j}^{u}$ \\
\hline MSCM & Modal curvatures $\phi^{\prime \prime}$ & $\sum_{j=1}^{m}\left|\varphi_{d, j}^{\prime \prime}-\varphi_{u, j}^{\prime \prime}\right|$ \\
\hline SCE & Modal curvatures $\phi^{\prime \prime}$ & $\Sigma^{m}\left|\varphi_{d, j}^{\prime \prime}-\varphi_{u, j}^{\prime \prime}\right|$ \\
\hline & & $\Sigma_{j=1}\left|\overline{\varphi_{u, j}^{\prime \prime}}\right|$ \\
\hline \multirow[t]{2}{*}{ DIM } & \multirow[t]{2}{*}{ Modal curvatures $\phi^{\prime \prime}$} & \multirow{2}{*}{$\beta_{i, j}=\frac{\left[\int_{a}^{D}\left(\varphi_{j}^{d \prime \prime}\right) d x+\int_{0}^{L}\left(\varphi_{j}^{d \prime \prime}\right) d x\right]}{\left[\int_{a}^{b}\left(\varphi_{j}^{u \prime \prime}\right)^{2} d x+\int_{0}^{L}\left(\varphi_{j}^{u \prime \prime}\right)^{2} d x\right]} \cdot \frac{\int_{0}^{L}\left(\varphi_{j}^{u \prime \prime}\right) d x}{\int_{0}^{L}\left(\varphi_{j}^{d \prime \prime}\right) d x}$} \\
\hline & & \\
\hline CFM & $\begin{array}{l}\text { Mass-scaled mode } \\
\text { shapes } \phi \text { or modal curvatures } \phi^{\prime \prime}\end{array}$ & $\beta=\operatorname{diag}\left\{\mathbf{F}^{d}-\mathbf{F}^{u}\right\} ; \mathbf{F}=\sum_{j=1}^{m} \frac{1}{\omega_{j}^{2}} \phi_{j} \phi_{j}^{T}$ \\
\hline
\end{tabular}

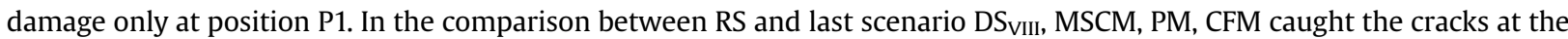
supports, SCE identified the damage at positions P1 and P9, the DIM indicated node P1 (only values higher than 2 are considered as damage in the DIM computation) and the COMAC barely detected the presence of damage at positions P2/P3 and P9/P10. Instead, in the relative comparison of $\mathrm{DS}_{\mathrm{VIII}}$ with the previous configuration, the COMAC resulted completely insensitive to damage, both DIM and SCE indicated node P11 as possible damage location, while MSCM, PM and CFM showed again peaks at positions P1 and P11.

Figs. 18 and 19 give a graphic insight into the results obtained from the different methods in terms of damage location and cracks sequence. It can be observed that no single method was able to locate all target nodes, except for the Power Spectral Density method. In fact, most of damage identification algorithms detected the presence of cracks at positions P1 and P11, but none of them attained a complete damage identification, probably because they were conceived for structures other than masonry constructions. The results obtained show then the robustness of the spectral formulation in detecting and locating the damage for the type of structure analysed and assert its potential suitability as a damage identification tool for masonry structures.

\section{Conclusions}

The present paper addresses a spectral-based method for non-destructive damage identification purposes. The fundamentals of the method are briefly reviewed and a simplified flowchart is presented to summarise the main steps of the technique, stressing the leading role of the eigenparameters extracted from the system's output power spectrum matrix in the damage investigation procedure. The case-study of an old masonry arch replica subjected to increasing damage is presented to evaluate the capability of the method to detect and locate the cracks at the earliest possible stage. It is observed that the occurrence and increase of damage can be easily caught by following the evolution of the eigenvalues over eight progressive damage scenarios, while the position of the cracks is pinpointed by computing an index based on the difference between spectral modes belonging to different structural conditions (e.g. damaged and undamaged). Relative comparisons between consecutive damage scenarios are carried out to explore the evolution of damage along the testing and the possible crack sequence. The use of two frequency bandwidths for the spectral algorithm showed the possibility to reduce the influence of noise in the modal data in order to achieve better outcomes.

A peculiar feature of the proposed spectral-based damage identification method is that it takes into account the contribution of both direct and cross-modal terms to PSDs. It is noted that if the damping in the system is small and the modal frequencies are well separated, then the contributions of cross-modal terms to auto spectral densities are smaller than the contributions from single modes given by direct-modal terms [11]. But if these conditions are not verified, the consideration of cross-modal terms is necessary. Since eigenvalues and eigenvectors already contain the cross-modal terms contributions, it follows that the analysis of the system's response PSD matrix through its eigenvalue decomposition can lead to more accurate results in terms of damage identification with respect to the analysis of single PSDs estimates [23].

In many years of publications about damage identification techniques, it has been shown that no method can be considered universally effective. However, the method hereby presented allowed to successfully locate the cracks in the structure under analysis at a very early stage, showing its potential suitability as a non-destructive damage identification 


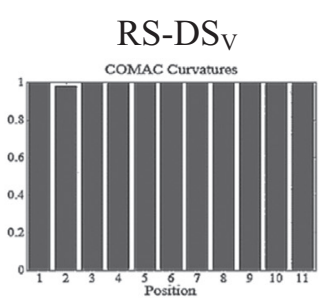

PM for Curvatures
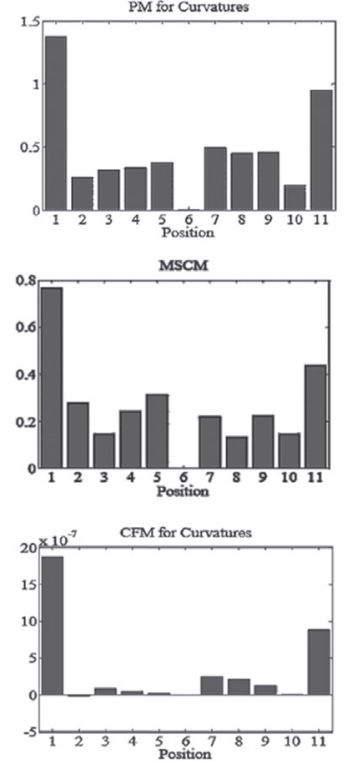

DIM

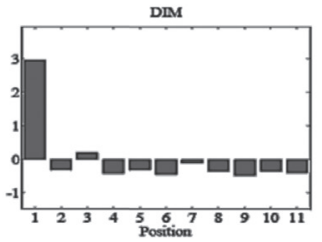

SCE

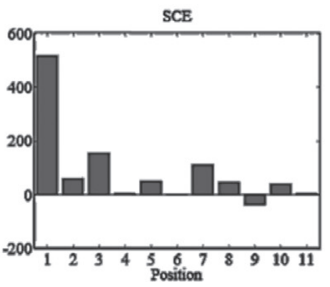

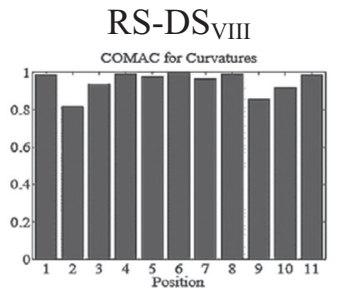

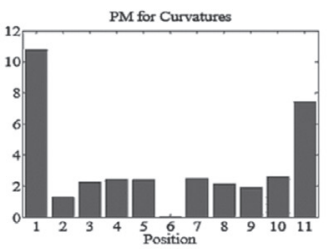

MSCM
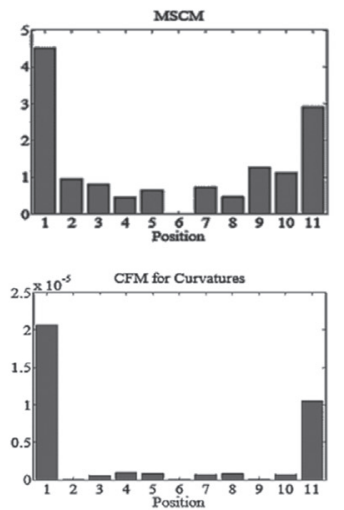

DIM

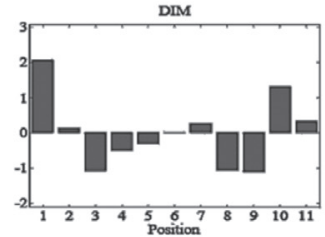

SCE

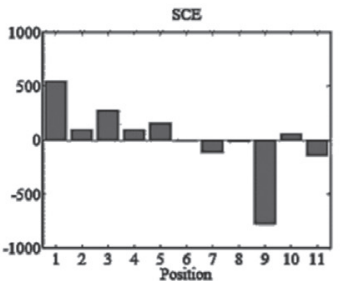

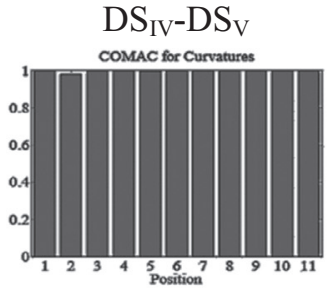

PM for Curvatures

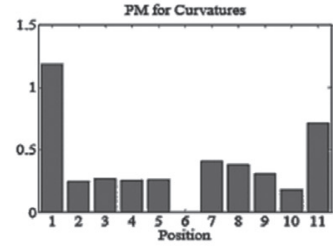

MSCM
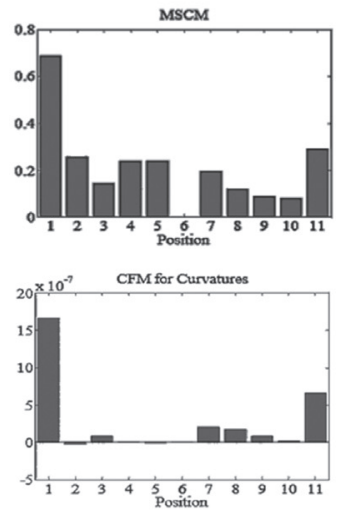

DIM

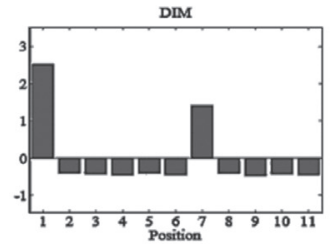

SCE

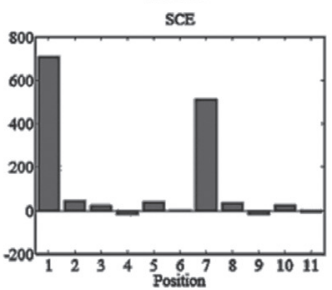

$\mathrm{DS}_{\mathrm{VII}}-\mathrm{DS}_{\mathrm{VIII}}$

COMAC for Curvatures

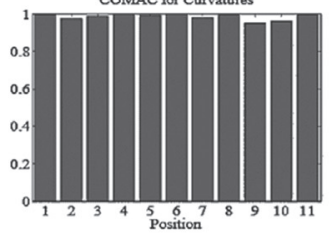

COMAC
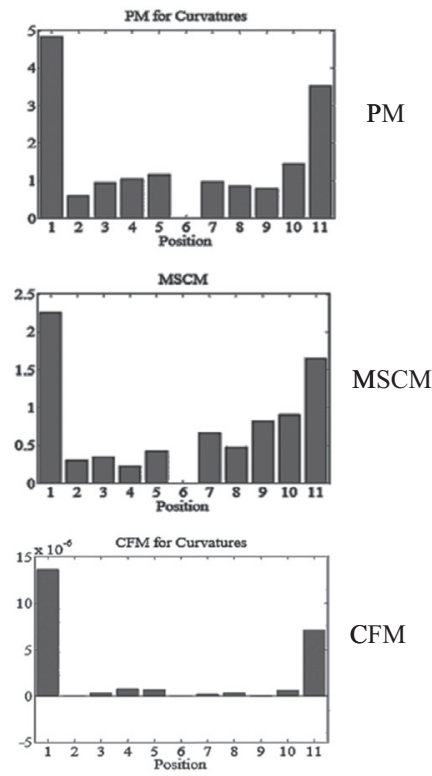

DIM

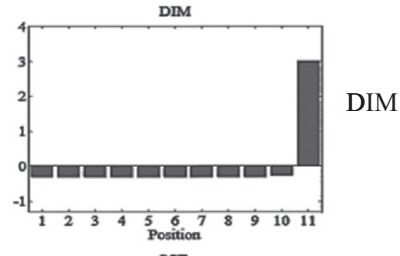

SCE

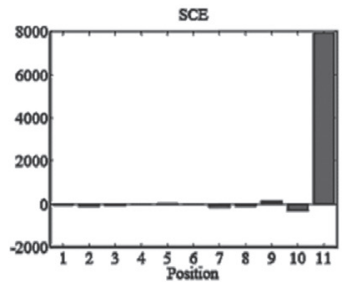

Fig. 17. Damage analysis results for measured curvatures by other vibration-based damage identification methods: comparison of $\mathrm{DS}_{\mathrm{V}}$ and $\mathrm{DS} \mathrm{VIII}_{\mathrm{I}}$ with the $\mathrm{RS}$ and with the previous configurations $\mathrm{DS}_{\mathrm{IV}}$ and $\mathrm{DS}_{\mathrm{VII}}$, respectively.

tool for masonry constructions. The spectral results proved to be satisfactory and in agreement with the experimental evidence, whereas most of the results obtained by other vibration-based damage identification methods applied to the same data sets turned out to be less accurate. Given the difficulty of the subject, further research is being carried out to better address important issues, such as the role of the spectral eigenvectors as spatial damage indicators and the efficiency of the spectral formulation when dealing with non-stationary stochastic processes. Another critical aspect to tackle is the application of the spectral-based method to real masonry structures. Due to the increased number of uncertainties and limitations in comparison with controlled experimental activities in lab environment, a straightforward application of the spectral algorithm might result difficult when dealing with masonry structures in operational conditions. Thus, issues related to optimal sensors location, type of modal analysis technique to adopt, data acquisition system, data processing and removal of environmental effects will be handled carefully in order to assess the performance of the developed formulation and address possible drawbacks. 


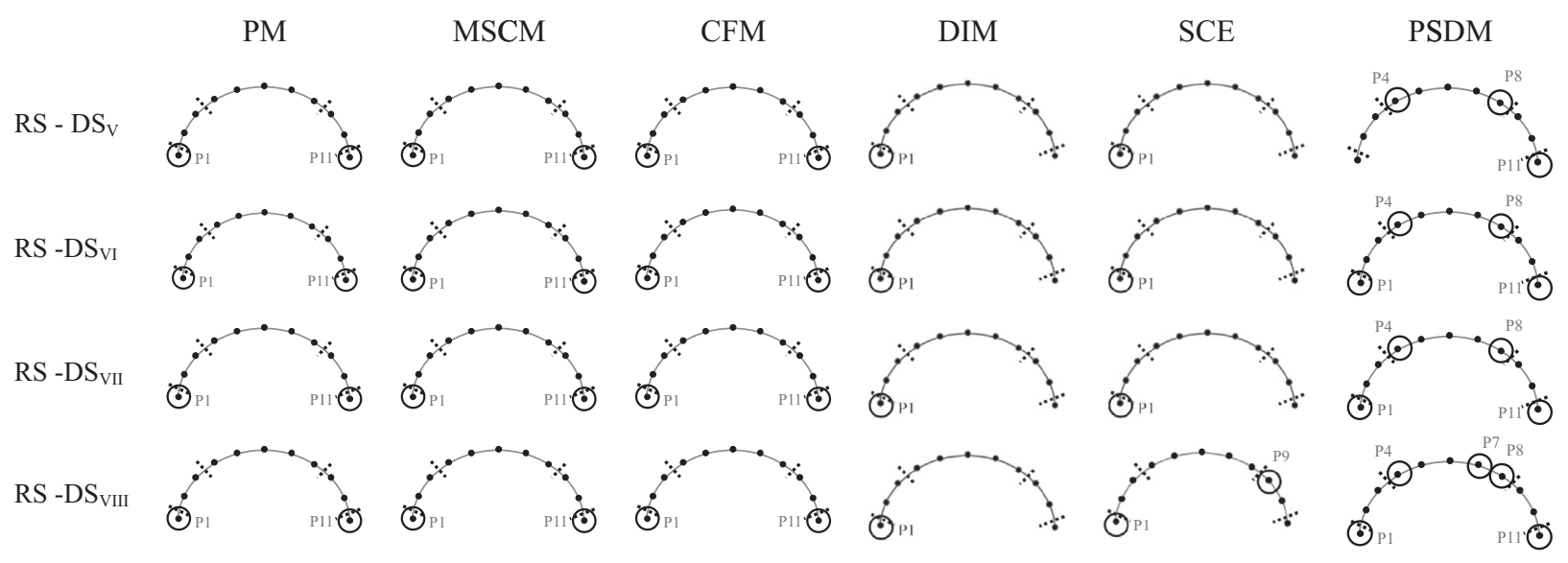

Fig. 18. Damage localisation and cracks sequence according to the VBDIMs specified in the columns header: comparison with the RS. The dotted lines indicate the observed cracks and the circles represent the cracks detected via VBDIMs.

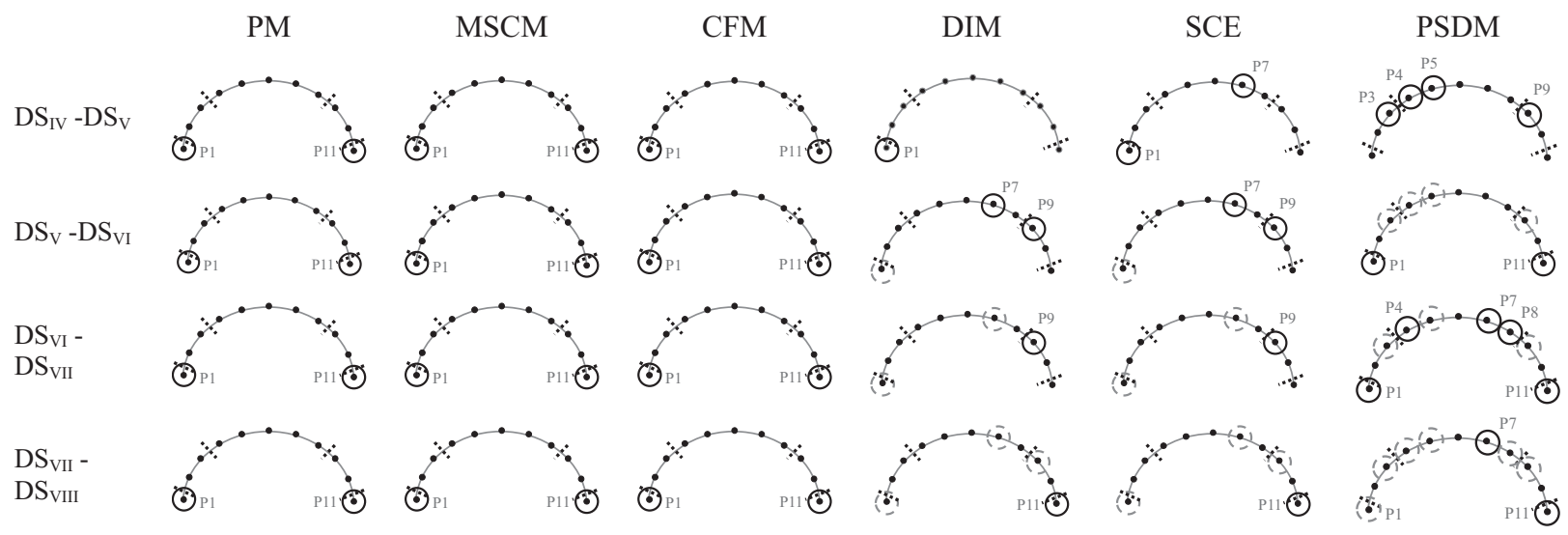

Fig. 19. Damage localisation and cracks sequence according to the VBDIMs specified in the columns header: comparison with each consecutive DS. The dotted lines indicate the observed cracks and the circles represent the cracks detected via VBDIMs.

\section{References}

[1] P.B. Lourenço, Recommendations for restoration of ancient buildings and the survival of a masonry chimney, Constr. Build. Mater. 20 (2006) $239-251$.

[2] M.M. Abdel Wahab, G. De Roeck, Damage detection in bridges using modal curvatures: application to a real damage scenario, J. Sound. Vib. 226 (1999) 217-235.

[3] O. Loland, C.J. Dodds, Experiences in developing and operating integrity monitoring systems in the north sea, in: Proceedings of the Eighth Annual Offshore Technology Conference, Houston, Texas, May 3-6, 1976, pp. 331-3319.

[4] R.D. Adams, et al., Vibration testing as a nondestructive test tool for composite materials, Composites 7 (1976) 203.

[5] C. Gentile, a Saisi, Ambient vibration testing of historic masonry towers for structural identification and damage assessment, Constr. Build. Mater. 21 (2007) 1311-1321.

[6] S. Doebling, C. Farrar, M. Prime, A summary review of vibration-based damage identification methods, Shock Vib. Dig. 30 (1998) $91-105$.

[7] S.-E. Fang, R. Perera, Power mode shapes for early damage detection in linear structures, J. Sound. Vib. 324 (2009) 40-56.

[8] S. Liberatore, G.P. Carman, Power spectral density analysis for damage identification and location, J. Sound. Vib. 274 (2004) $761-776$.

[9] L.F. Ramos, G. De Roeck, P.B. Lourenço, A. Campos-Costa, Damage identification on arched masonry structures using ambient and random impact vibrations, Eng. Struct. 32 (2010) 146-162.

[10] Y.K. Lin, Probabilistic Theory of Structural Dynamics, Krieger, Malabar FL, 1986.

[11] L. Lutes, S. Sarkani, Random Vibrations: Analysis of Structural and Mechanical Systems, Butterworth-Heinemann, MA, USA, 2004.

[12] L.H. Yam, Y.J. Yan, Z. Wei, Vibration-based non-destructive structural damage detection, Key Eng. Mater. 270-273 (2004) 1446-1453.

[13] A. Rytter, Vibrational Based Inspection of Civil Engineering Structures (Ph.D Thesis), University of Aalborg, Denmark, 1993.

[14] K. Worden, J.M. Dulieu-Barton, An overview of intelligent fault detection in systems and structures, Struct. Health Monit. 3 (2004) 85-98.

[15] M. Richardson, Is it a mode shape, or an operating deflection shape?, Sound and Vibration Magazine 30th Anniversary Issue, 1997.

[16] L.F. Ramos, Damage Identification on Masonry Structures Based on Vibration Signatures (Ph.D Thesis), University of Minho, Portugal, 2007.

[17] I. Basilio, Strengthening of Arched Masonry Structures With Composite Materials (Ph.D Thesis), University of Minho, Portugal, 2007.

[19] B. Peeters, G. De Roeck, Reference-based stochastic subspace identification for output-only modal analysis, Mech. Syst. Signal Process. 13 (1999) 855-878.

[20] R.W. Clough, J. Penzien, Dynamics of Structures, 2nd edition, . McGraw-Hill International Editions, New York, 1993.

[21] C.B. Moler, Eigenvalues and singular values, in numerical computing with MATLAB, Soc. Ind. Appl. Math. (2004) 269-305. ch. 10.

[22] M. Di Paola, Digital simulation of wind field velocity, J. Wind Eng. Ind. Aerodyn. 74-76 (1998) 91-109. 
[23] M.-G. Masciotta, L.F. Ramos, P.B. Lourenço, M. Vasta, G. De Roeck, A spectrum-driven damage identification technique: application and validation through the numerical simulation of the Z24 Bridge, Mech. Syst. Signal Process. 70-71 (2016) 578-600.

[24] R. Cantieni, Office floor vibrations: modal parameter identification and vibration monitoring, in: Proceedings of ISMA 2006, Leuven, September 18-20, pp. 2937-2948.

[25] E. Reynders, G. De Roeck, Reference-Based Combined Deterministic-Stochastic Subspace, in: Proceedings of ISMA 2006, Leuven, September 18-20, pp. 3035-3046. 